\title{
Identification and analysis of transport domains in the stochastic boundary of TEXTOR-DED for different mode spectra
}

\author{
O. Schmitz ${ }^{1}$, , M. W. Jakubowski ${ }^{4}$, H. Frerichs ${ }^{1}$, D. Harting ${ }^{1}$, \\ M. Lehnen ${ }^{1}$, B. Unterberg ${ }^{1}$, S.S Abduallaev ${ }^{1}$, S. Brezinsek ${ }^{1}$, I. \\ Classen $^{2}$, T. Evans ${ }^{3}$, Y. Feng ${ }^{4}$, K. H. Finken ${ }^{1}$, M. Kantor ${ }^{1}$, D. \\ Reiter $^{1}$, U. Samm ${ }^{1}$, B. Schweer ${ }^{1}$, G. Sergienko ${ }^{1}$, G. W. \\ Spakman $^{2}$, M.Tokar ${ }^{1}$, E. Uzgel ${ }^{1}$, R. C. Wolf ${ }^{1}$ and the TEXTOR \\ Team \\ ${ }^{1}$ Institut für Energieforschung - Plasmaphysik, Forschungszentrum Jülich GmbH in \\ der Helmhotz-Gemeinschaft, Association EURATOM-FZJ, Trilateral Euregio \\ Cluster, D-52425 Jülich, Germany \\ ${ }^{2}$ FOM-Institute for Plasma Physics Rijnhuizen, Trilateral Euregio Cluster, \\ Association EURATOM-FOM, PO-Box 1207, 3430 BE Nieuwegein, The Netherlands \\ ${ }^{3}$ General Atomics, San Diego, California, USA \\ ${ }^{4}$ Max-Planck-Institut für Plasmaphysik, EURATOM Association, 17491 Greifswald, \\ Germany \\ E-mail: o.schmitz@fz-juelich.de
}

\begin{abstract}
At the TEXTOR tokamak an external resonant magnetic perturbation is applied with the Dynamic Ergodic Divertor to control the edge transport properties. The approaches to analyze the impact of such kind of edge stochastisation on transport apply mostly a shell like picture which includes a dependence of transport from magnetic field topology in the radial direction only. In this paper multiple experimental evidence is presented that contrary to these approaches the perturbation applied forms a poloidally heterogenous edge layer in which the transport characteristics are determined by the poloidally alternating field line behavior. A thorough analysis of density and temperature profiles and their gradients for base mode spectra with poloidal/toroidal mode numbers of $m / n=12 / 4$ and $m / n=6 / 2$ is worked out in comparison to the modeled magnetic field topology and results from three dimensional transport modeling with EMC3/EIRENE. Hereby two poloidally adjacent transport domains are identified for the first time in such detail. A domain representing a helical scrape off layer $(S O L)$ is formed by field lines with short connection and therefore prevailing parallel transport to the wall elements. Here, the field lines are clustered into extended flux tubes embedded into a long connection length ergodic domain with diffusive transport characteristics and enhanced radial transport.
\end{abstract}

PACS numbers: 28.52.Av, 52.25.Fi, 52.40.Hf, 52.55.Dy, 52.55.Rk, 52.65.Pp 


\section{Introduction}

The controlled exhaust of particles and energy is one key topic for next step fusion devices as the tokamak ITER or the stellarator $W \%-X$. In this context perturbations of the magnetic equilibrium forming a stochastic magnetic boundary are in the actual focus of interest.

In stellarators magnetic islands and stochastic magnetic boundaries exist due to the non axissymetric magnetic field geometry and additional error fields from the confining magnetic coil system or external magnetic perturbations respectively. The resulting three dimensional magnetic field topology is utilized in the helical divertor $[1,2]$ and the island divertor [3] concept to control particle and heat exhaust. In addition, a more dedicated edge control system is created in the local island divertor [4] concept by the formation of magnetic islands with external magnetic fields. Within these concepts magnetic islands and stochastic magnetic boundaries are the basis for particle and heat exhaust in stellarators.

In high confinement mode (H-mode) plasmas of poloidal divertor tokamaks stochastic magnetic boundaries are of growing importance for edge transport control. H-mode plasmas are characterized by steep edge pressure gradients and associated edge instabilities, causing a repetitive relaxation of over-critical edge pressure gradients - the so called edge localized modes (ELMs)[5]. They lead to a short timed (order of $100 \mu \mathrm{s}$ ) heat pulse which brings up to $20 \%$ of the stored energy to the divertor target. The energy load of this so called type-I ELMs will be beyond the technical limits of the wall components in ITER, causing e.g. carbon ablation and a significant reduction of the wall lifetime [6]. The mitigation of type-I ELMs is therefore one of the physical key topics for ITER. It was successfully demonstrated that such ELMs can be mitigated or even completely suppressed with externally applied, non-axissymetric, resonant magnetic perturbations (RMPs) while maintaining the H-mode performance $[7,8]$. These RMPs create a stochastic magnetic boundary which gives control on the edge transport properties and by that suppressing ELMs reliably in ITER similar shapes and collisionality conditions [9]. However, the generic physical mechanisms of this promising approach are still under investigation and a thorough understanding of transport in stochastic magnetic fields is necessary to design a RMP coil system for ITER.

At the TEXTOR tokamak the Dynamic Ergodic Divertor (DED) was designed as a flexible setup to investigate the generic features of stochastic magnetic boundaries [10]. As visualized in figure 1 it consists of a set of $16+2$ coils implemented into the vacuum vessel and winding helically around the torus at the high field side (HFS). They create a spectrum with main resonances around the $q=3$ surface and poloidal/toroidal base mode numbers of $m / n=3 / 1,6 / 2,12 / 4$ can be selected by different wiring of the perturbation coils. The fields induced can be rotated with frequencies of $\nu=1-10 \mathrm{kHz}$. To facilitate the experimental investigation the magnetic topology induced can be moved by low frequency $(\nu=1,2 \mathrm{~Hz})$ strike point sweeping. 
In this paper the results of a detailed investigation of the plasma structure and the correlated transport characteristics in the DED induced stochastic edge layer are presented. The aim was to resolve experimentally the three dimensional structure of the stochastic boundary and to identify basic transport mechanisms by comparison to appropriate numerical tools. The experimental results then allow to validate these codes and to generalize the findings at TEXTOR-DED in order contribute with these results to the understanding of the mechanisms of ELM control in tokamaks and the exhaust concepts of stellarators. Therefore a thorough analysis of measurements in poloidal and in radial direction at different toroidal positions was worked out in $m / n=12 / 4$ and $m / n=6 / 2$ base mode configuration.

For this task the following experimental and numerical tools were adapted and subsequently applied. On the experimental side spatially high resolving edge diagnostics were utilized: to measure the electron density $n_{e}$ and the electron temperature $T_{e}$ active beam emission spectroscopy (BES) on thermal helium beams was applied as the basic diagnostic. These radial measurements were conducted at two toroidal positions in the equatorial midplane at high field side (HFS, poloidal angle $\vartheta=185 \mathrm{deg}$ ) and at low field side (LFS, $\vartheta=5 \mathrm{deg}$ ) of the TEXTOR vessel [11]. In addition a new edge Thomson scattering system [12] was used to measure $n_{e}(r)$ and $T_{e}(r)$ at the poloidally upper position of the vessel $(\vartheta=280 \mathrm{deg})$. Both diagnostics have a high spatial resolution of $\Delta r \sim 1 \mathrm{~mm}$. Therefore they allow us to deduce gradients and by that to investigate the induced transport characteristics in the stochastic edge layer of TEXTOR-DED. These profile measurements were accompanied by techniques with two dimensional spatial resolution. The time evolution of $T_{e}(r, \vartheta)$ was measured with an electron cyclotron emission imaging (ECE-I) system [13, 14]. With an array of $16 \times 8$ channels an observation area of $9 \mathrm{~cm}$ radially and $30 \mathrm{deg}$ poloidally is covered allowing simultaneous two dimensional observations of $T_{e}(r, \vartheta)$ in this area. These measurements were completed by CCD cameras equipped with $H_{\alpha}\left(\lambda_{H_{\alpha}}=656.6 \mathrm{~nm}, \Delta_{F W H M}=50 \mathrm{~nm}\right)$ and $C I I I\left(\lambda_{C I I I}=456.6 \mathrm{~nm}, \Delta_{F W H M}=5 \mathrm{~nm}\right)$ interference filters and with viewing chords tangentially along and perpendicular to the DED-target. Right part of figure 1 shows as an example for the tangentially observed CIII emission in the perturbed edge layer.

As numerical tools for magnetic modeling two codes employing the hamiltonian formalism to calculate the field line trajectories were applied. The ATLAS code [15] a simplectic mapping code for magnetic field lines - and the GOURDON code [16] a numerical field line tracing code - were used and validated by comparison to the experimental results. Both approaches use a vacuum approximation - i.e. a linear superposition of the equilibrium magnetic field and the external perturbation field - and one crucial question is if this approach is sufficient for a proper magnetic modeling. For a comparative transport modeling in the complex, three dimensional stochastic edge layer the EMC3/EIRENE code [17] was applied subsequently. This fully three-dimensional 
Monte Carlo code solves the Braginskii fluid equations (EMC3) taking into account the particle, energy and momentum sources and sinks in front of and on the wall (EIRENE). Hereby, it is a unique tool to model three dimensional edge plasmas in stellarators as well as in tokamaks.

This paper is organized as follows: first the magnetic topology of the stochastic edge layer is introduced and compared in detail to the experimental observations. On this basis the identification of the characteristic transport domains of the stochastic edge layer is presented. These findings will be accompanied by comparison to results from EMC3/EIRENE modeling. This start of a quantitative validation will enable us in future to relate the findings from TEXTOR-DED for analysis of results from advanced experiments as DIII-D and JET and to use the code as a predictive tool for ITER and W7-X application. This paper will end with an corresponding outlook.

\section{Magnetic topology and its expected relation to transport}

The task to understand the transport properties in stochastic edge layers is a long term project in modern plasma physics. It was stated e.g. in 1978 that braiding of the magnetic field shall lead to an enhanced radial heat transport [18] which encouraged plasma physicists to explore in how far this could be utilized for a reduction of the localized heat deposition on the wall elements. First dedicated experimental approaches with resonant magnetic perturbation $(R M P)$ coils were conducted at several experiments as TEXT (USA) [19], JIPP T-IIU (Japan)[20] and at Tore Supra (France) [21] for example. They demonstrated that the transport in the plasma edge can be controlled with RMPs and that the link between magnetic topology on the one hand and the transport characteristics on the other hand is of fundamental importance. According to this context the DED was designed for TEXTOR and the ATLAS and GOURDON codes as tools for magnetic modeling were developed and adapted respectively. They allow fast calculations of the magnetic field topology and in combination with the advanced edge diagnostic equipment at TEXTOR detailed comparisons to experimental findings in both, the radial and the poloidal direction became possible.

Figure 2 shows an example of the magnetic topology as combination of a Poincaré plot and a so called laminar plot modeled for a typical TEXTOR discharge in $m / n=6 / 2$ base mode (plasma current $I_{p}=365 k A$, toroidal magnetic field $B_{t}=1.9 \mathrm{~T}$, edge safety factor $q_{a}=3.6$, total heating power $P_{\text {tot }}=600 \mathrm{~kW}$, poloidal beta $\beta_{\text {pol }} \simeq 0.3$, central plasma position $R_{0}=1.73 \mathrm{~m}$ and minor radius $a=0.46 \mathrm{~m}$ ). The Poincaré plot shows the intersections of the magnetic field lines with the selected poloidal plane and exhibits the stochastic behavior of the field lines. The laminar plot quantifies the connection length $L_{c}$ of the field lines as field line length from target to target in units of meters $[m]$ or poloidal turns $[$ p.t. $]$ respectively. $L_{c}$ is depicted as a color coded contour plot. These two techniques then give a detailed picture of the magnetic topology and quantify 
$L_{c}$ as one basic transport related feature of the topology induced.

In figure 2 it is demonstrated that this topology consists out of three regions with very different transport characteristics to be expected. Starting at small radii, magnetic islands are created in the region of $r / a \simeq 0.75$ (i.e. $\mathrm{r} \simeq 0.35 \mathrm{~m}$ ). The islands' size grows on flux surfaces closer to the perturbation source and defined by field lines with mode numbers resonant to the high amplitude fourier components of the RMP field. The island chains of neighboring resonant flux surfaces overlap and the field lines here get stochastic. They are not bound to the flux surface or single island chains anymore but become highly diffusive filling the whole volume in radial and poloidal direction also. This behavior is termed ergodic and this region is therefore called the ergodic region. However, as the RMP coils are located at the high field side (HFS) the field lines of the outer edge will be diverted towards the DED target. These near field effects induce an extended region filled with short $L_{c}$ field lines, the so called laminar zone [16, 22]. The field lines here are bundled into short $L_{c}$ flux tubes with an extension of up to $2-4 \mathrm{~cm}$ in radial and up to $10 \mathrm{~cm}$ in poloidal direction. These values are an example in $m / n=12 / 4$ base mode configuration and they are increased to a more coarse structure in the base mode configurations with lower mode numbers $(m / n=6 / 2,3 / 1)$. Figure 3 shows a direct comparison of the magnetic topology in $m / n=12 / 4$ base mode (left figure) and in $m / n=6 / 2$ base mode (right figure) as these two operation modes are the subject of investigation for this paper. The laminar zone in $m / n=6 / 2$ configuration is nearly as large as the whole stochastic volume in $m / n=12 / 4$ configuration and the laminar flux tubes extend up to $3-6 \mathrm{~cm}$ in radial and $15-30 \mathrm{~cm}$ in poloidal direction. The consequences of these topological features for transport characteristics are discussed in this paper.

In summary the DED induced stochastic edge layer typically consists of a mixture of ergodic field lines with long $L_{c}>50 \mathrm{~m}$ (red, blue, green and light green regions in figure 2 ) and extended flux tubes with short $L_{c}$ field lines, connecting within $20-30 \mathrm{~m}$ directly to the DED target (orange and yellow areas in figure 2). The basic question for the understanding of the transport processes in such kind of stochastic edge layers is how these very different topological domains are reflected in the plasma and what effect on transport properties they exhibit.

The field lines' connection length is only one measure aiming at a thorough transport analysis in stochastic edge layers. In order to quantify magnetic field stochastisation commonly three poloidally averaged parameters are used [23]. The Chirikov parameter $\sigma_{\text {chir }}$ describes the ratio of the island width of the RMP induced island chains on neighboring resonant surfaces and the distance of these flux surfaces. It becomes larger than one when the islands overlap and this magnetic flux region is termed as ergodic. Another measure is the Kolmogorov length $L_{K}$ which quantifies the typical length of de-correlation along magnetic field lines. Applying $L_{K}$ as a measure for ergodicity, a volume becomes ergodic as soon as $L_{K} \ll L_{c}$. Finally the diffusivity of the field lines 
in a stochastic magnetic field is described by the field line diffusion coefficient $D_{f l}$. The later two quantities are derived with the ATLAS code as so called local values by a statistical evaluation of the field line behavior during the mapping procedure $[23,24]$. However, in existing approaches these quantities are functions of the radial coordinate only.

Figure 4 shows the profiles of $D_{f l}(r)$ (dashed blue curve, left ordinate) and of $L_{K}(r)$ (solid green curve, right ordinate) for $m / n=12 / 4$ base mode (upper figure) and for $m / n=6 / 2$ base mode (lower figure). These calculations were performed with the ATLAS code for a comparative perturbation current. The area where $\sigma_{\text {chir }} \geq 1$ is shadowed with grey boxes and marked with the red arrow depicting the radial extension of the stochastic edge. Based on the $D_{f l}(r)$ profile the ergodic zone can be distinguished from the laminar zone as here $D_{f l}$ starts to decrease. The reason is that in the laminar zone with increasing radius more and more field lines connect to the wall. This results in a loss of field lines which do not perform the full amount of numerical turns in the mean averaged evaluation of the field line behavior. Therefore $D_{f l}$ decreases if a field line starts in the the laminar zone. The grey shadowing accordingly distinguishes the ergodic from the laminar zone.

The results shown in figure 4 add two information to the laminar plot visualization discussed before. First it confirms the larger radial extension of the whole stochastic edge in $m / n=6 / 2$ base mode $\left(\Delta r_{\text {stoch }} \simeq 8 \mathrm{~cm}\right)$ compared to $m / n=12 / 4$ base mode $\left(\Delta r_{\text {stoch }} \simeq 4 \mathrm{~cm}\right)$. Second the maximum $D_{f l}=7 \times 10^{-6} \mathrm{~m}$ in $\mathrm{m} / \mathrm{n}=6 / 2$ base mode is more than twice as high as in $m / n=12 / 4$ base mode $\left(D_{f l}=2.5 \times 10^{-6} \mathrm{~m}\right)$. Applying these poloidally averaged quantities in existing concepts for transport in stochastic fields (e.g. $[18,21,25,26]$ ) a significant enhancement of radial heat conductivity as well as of the radial particle diffusion coefficient would be expected. Depending on the model applied, changes of these transport coefficients by a factor of 2 up to 30 would be anticipated in the whole stochastic edge region of DED [17].

However, in contrast to these poloidally averaged approaches the laminar plot visualization showed that the DED induced edge layer is highly asymmetric in this coordinate direction and therefore the transport properties shall be too. It is supposed that in the short $L_{c}$ flux tubes the fast transport along the magnetic field lines to the DED target will open a well defined sink structure into a region of formerly enclosed flux surfaces. Therefore these flux tubes can potentially act as a multipolar, helical scrape off layer (SOL). This suggests that during comparison of experimental results to the predictions by 1-D approaches the exact location of the diagnostic in the 3D magnetic topology is of fundamental importance. The transport impact based on $\sigma_{c h i r}(r), L_{K}(r)$ and $D_{f l}(r)$ shall be valid in the ergodic domain with long $L_{c}$. Here an enhanced radial transport is expected, likely to be in correspondence to 1-D description. First experimental and theoretical evidence for these facts was presented already from the experiments at Tore Supra [21, 22]. However, the combination of sophisticated magnetic modeling tools with an advanced edge diagnostic equipment with high spatial 
resolution in both, radial and poloidal direction, allowed to identify these contrary transport channels and to resolve the correlated mechanisms in the stochastic edge layer of TEXTOR-DED for the first time in very detail [27, 28, 29, 30, 31, 32]. An extension of these results for two different base mode spectra and an overview discussion of the transport characteristics supported by EMC3/EIRENE modeling is presented in this paper.

\section{Experimental identification of the transport domains}

The identification of transport domains in the DED induced stochastic boundary is based on direct comparisons of experimental data with the calculated magnetic topology. The following exploration will start with an evaluation of two dimensional measurements of the electron temperature $T_{e}(r, \vartheta)$ and density $n_{e}(r, \vartheta)$ field in the stochastic edge for $m / n=6 / 2$ and $m / n=12 / 4$ base mode configuration followed by a thorough analysis of radial $T_{e}(r)$ and $n_{e}(r)$ profiles and their gradients $\nabla T_{e}(r)$ and $\nabla n_{e}(r)$.

Figure 5 shows the analysis of results from ECE-I measurements for a typical discharge scenario in $m / n=6 / 2$ base mode configuration (plasma current $I_{p}=365 \mathrm{kA}$, toroidal magnetic field $B_{t}=1.9 \mathrm{~T}$, edge safety factor $q_{a}=3.6$, total heating power $P_{\text {tot }}=600 \mathrm{~kW}$, poloidal beta $\beta_{\text {pol }} \simeq 0.3$, central plasma position $R_{0}=1.73 \mathrm{~m}$ and minor radius $a=0.458 \mathrm{~m})$. Plots on the right side show the measured $\hat{T}_{e}(r, \vartheta)$ distribution, i.e. $T_{e}(r, \vartheta)$ normalized to a reference point without perturbation in the same discharge. On the left side the modeled connection length distribution $L_{c}(r, \vartheta)$ is depicted. The upper row of the figure shows this set for a discharge with standard $I_{P}$ and $B_{t}$ direction (TEXTOR \#101435) while the lower row shows the result for a discharge in reversed $I_{P}$ and $B_{t}$ configuration (TEXTOR \#101431). The reversal of both parameters leads to a shift of the induced topology by $180 / n$ deg ( $\mathrm{n}$ is the toroidal mode number) in toroidal direction as the plasma's poloidal magnetic field component reverses its direction relative to the perturbation field $B_{r}(r, \vartheta)$. Due to the helical perturbation field this toroidal shift leads at a fixed measurement position to a poloidal shift. This becomes obvious comparing the modeled $L_{c}(\vartheta, r)$ distribution in figure 5 for both discharge setups. The ergodic region with long $L_{c}$ (red colored area) and the laminar flux tube with short $L_{c}$ (blue colored area) exchange their position.

The measured $\hat{T}_{e}(r, \vartheta)$ distribution for these discharges clearly exhibits a poloidal modulation of the electron temperature field closely correlated to the magnetic topology. In the vicinity of short $L_{c}$ the electron temperature $T_{e}$ is strongly reduced. Here we measure $\hat{T}_{e} \simeq 0.7$ at $r \simeq 0.42 \mathrm{~m}$ and in between $\vartheta \simeq 0-15 \mathrm{deg}$ as shown in the upper plot of figure 5 . For the reversed field and plasma current operation we get the same strong reduction, but now at $r \simeq 0.42 \mathrm{~m}$ and $\vartheta \simeq 30-45 \mathrm{deg}$ as depicted in the lower plot of figure 5 . In the ergodic region $\hat{T}_{e}$ is only marginally affected as we measure $\hat{T}_{e} \geq 0.9$ at $r \simeq 0.41 \mathrm{~m}$ and $\vartheta \simeq 0-25 \mathrm{deg}$ (lower plot in figure 5). For the standard field and plasma current we get this nearly unchanged $T_{e}$ value at $r \simeq 0.41 \mathrm{~m}$ and in 
between $\vartheta \simeq 30-45 \mathrm{deg}$ visualized again in the upper plot of figure 5 . The shift of the magnetic topology leads to a correspondent change in $\hat{T}_{e}(r, \vartheta)$ as demonstrated by these two dimensional measurements. They are therefore a direct proof of the poloidal asymmetry in the electron temperature field of the DED induced stochastic edge layer.

This initial information was extended by measurements of the $n_{e}(r, \vartheta)$ and $T_{e}(r, \vartheta)$ fields with the He beam diagnostic at the LFS in $m / n=12 / 4$ base mode configuration (TEXTOR \#95924 with $I_{p}=365 k A, B_{t}=1.9 T, q_{a}=3.6, P_{t o t}=1200 \mathrm{~kW}, \beta_{\text {pol }} \simeq 0.5$, $R_{0}=1.73 \mathrm{~m}$ and $\left.a=0.459 \mathrm{~m}\right)$. Using the strike point sweep mode of the DED the magnetic topology is shifted by $\Delta \vartheta=15^{\circ}$ at LFS. This allows to measure radial $n_{e}(r)$ and $T_{e}(r)$ profiles in the short $L_{c}$ flux tubes as well as in the adjacent ergodic regions $[28,32]$. The calculated magnetic topology is not modified but evenly shifted during the sweeps and it could therefore be correlated to the time evolution of $n_{e}(r, t)$ and $T_{e}(r, t)$. Accordingly a transformation into a radial and poloidal temperature and density field $n_{e}(r, \vartheta)$ and $T_{e}(r, \vartheta)$ was performed.

In figure 6 the correlation of the connection lengths distribution $L_{c}(r, \vartheta)$ - convoluted with the beam divergence - with $n_{e}(r, \vartheta)$ (upper right) and $T_{e}(r, \vartheta)$ (lower right) is visualized. To support visual comparison the contours of the color coded $L_{c}(r, \vartheta)$ distribution from left figure are superimposed in the $n_{e}(r, \vartheta)$ and $T_{e}(r, \vartheta)$ figures on the right. This analysis clearly exhibits that field lines with short $L_{c}$ lead to a decrease of $n_{e}$ and $T_{e}$. In the adjacent regions with longer $L_{c}$ field lines both $n_{e}$ and $T_{e}$ are higher. Obviously the short $L_{c}$ region open a sink towards the DED target in a region of formerly closed flux surfaces (position of the last closed flux surface (LCFS) without RMP field is $r=0.443 \mathrm{~m}$ in the radial coordinate of the He beam diagnostic used here). These results prove an extension and restructuring of the scrape off layer (SOL) represented by the short $L_{c}$ flux tubes during RMP application. The SOL is not anymore a poloidally homogenous shell of short field lines but the stochastic edge layer consists of short $L_{c}$ flux tubes interleaved with ergodic regions. This was predicted by magnetic modeling [15], two dimensional [16] and three dimensional [17] modeling and the experimental results presented here prove that the plasma reflects these structures.

This good agreement of plasma structure and modeled magnetic topology for $m / n=$ $12 / 4$ and $m / n=6 / 2$ base mode configuration proves that for these base modes the vacuum approximation applied holds in the investigated radial domain. Obviously a screening of the perturbation field does not take place in the radial region of the island chains dominant for the formation of the laminar zone. As the perturbation field penetrates only in the colder edge region with weak conductivity screening currents seem to be weak and the calculated topology is in good correspondence to the plasma structures observed. This finding, supported by detailed comparisons of the spectroscopically extracted emission pattern on and in front of the DED target (see right image in figure 1 as example and references $[28,33,30]$ ), is therefore the basis for the following more detailed transport analysis. 
As first approach to compare particle and energy transport in the stochastic edge layer, we point out that the reduction of $n_{e}(r, \vartheta)$ and $T_{e}(r, \vartheta)$ shown in figure 6 is different. The $n_{e}$ value with perturbation normalized to the value of the unperturbed edge is $\hat{n_{e}}=0.5$ while $\hat{T}_{e}=0.7$ inside of the laminar flux tube. Inside of the ergodic domain $\hat{n_{e}}=0.7$ and $\hat{T}_{e}=0.85$. We emphasize here that these values are normalized values and therefore no matter of measurement accuracy. With the He beam diagnostic $n_{e}$ and $T_{e}$ are measured with a typical systematic error of $\Delta n_{e} \approx 10 \%$ and $\Delta T_{e} \approx 30 \%$ resulting from the atomic model used [11]. In the range of the parameter variation discussed here no change of this systematic uncertainties in measurement is expected. Therefore the deviation between $\hat{n}_{e}$ and $\hat{T}_{e}$ are reliable numbers. This means that we observe experimentally at TEXTOR-DED a strong influence on $n_{e}$ and a weaker manipulation of $T_{e}$. The hypothesis of enhanced radial energy transport can not be validated here but a strong impact on the particle transport is observed. To resolve the underlying transport mechanisms a careful evaluation of the gradients of the measured edge profiles was performed.

The results are shown in figure 7 . In the upper plots the $n_{e}(r)$ (left side) and $T_{e}(r)$ (right side) profiles are depicted for three time windows of the discharge: in red the profile without RMP, in green the phase during strike point sweep when the ergodic region with long $L_{c}$ field lines dominates the measurement volume (dashed line 1 in figure 6) and in blue the profiles when the short $L_{c}$ flux tube channels particles and energy towards the DED target (dashed line 2 in figure 6). In the lower plots the correspondent gradient profiles $\nabla n_{e}(r)$ (left) and $\nabla T_{e}(r)$ (right) are shown.

Focusing first on the density profiles it becomes obvious that $\nabla n_{e}(r)$ is slightly reduced comparing unperturbed (red profile) and ergodic (green profile) phase. The slope of the latter gradient profile does not change in general but the value, especially in the radial region were the $\nabla n_{e}$ runs towards a constant value $(r=0.42-0.43 \mathrm{~m})$, is reduced by about $\sim 20 \%$. This observation is discussed here in a simple diffusive picture where the radial particle flux is defined by $\nabla n_{e}$ and the radial particle diffusion coefficient $D_{\perp}$ in $\Gamma_{\perp}=D_{\perp} \cdot \nabla n_{e}$. In this simple picture we neglect anomalous transport effects and manipulation of the particle sources. Hereto the radial particle flux $\Gamma_{\perp}$ into this volume was assumed as constant based on the analyzed recycling behavior in front of the DED target. The particles recycle for this base mode to about $70 \%$ into the long $L_{c}$ domains which connect to a volume radially inside of the the evaluated region at the LFS [30]. Under this simplifying assumptions the slight change of $20 \%$ in $\nabla n_{e}$ means an enhancement of the radial particle transport - parametrised in $D_{\perp}$ - on the same level. In this base mode configuration the radial particle transport is slightly increased by at most $20 \%$ in the ergodic regions only. Obviously in this domain parallel transport to the wall elements is negligible, even though there is a small fraction of short field lines embedded into the observation volume. The long $L_{c}$ field lines determine the transport behavior in this ergodic domain leading to a diffusive radial transport characteristics. 
In contrast the gradients $\nabla n_{e}$ are much stronger reduced in the flux tube dominated phase of the discharge. However, the previous discussion pointed out that in this vicinity the direct parallel transport to the DED target dominates. This means that due to the fast transport along the field lines the sink functionality of the flux tubes makes the profiles - and by that the gradients as well - decaying exponentially. This behavior is clearly exhibited by this detailed experimental results distinguishing the SOL flux tubes as a domain of parallel, convective transport from the diffusive ergodic regions. Here the radial transport has diffusive character and is slightly enhanced acting as particle and energy source region for the multipolar SOL represented by the short $L_{c}$ flux tubes. Referring to the idea of classical ergodic transport [18] the strongest impact was expected for radial heat transport. With the radial heat conductivity $\kappa_{\perp}$ the radial heat flux is defined by $q_{\perp}=-\kappa_{\perp} \cdot \nabla T_{e}+5 / 2 \Gamma_{\perp} \cdot T_{e}$. Examining the $\nabla T_{e}$ profiles in the lower right plot of figure 7 nearly no change in $\nabla T_{e}$ is observed, not even in the experimentally identified ergodic regions. This is in contradiction to the classical picture on ergodic transport. It means that the heat loss which occurs is obviously convective caused by the particle fluxes inside of the SOL flux tubes.

Sweeping the DED currents limits their amplitude to half of the maximum value in DC operation [32]. Therefore the finding discussed before was confirmed by an experiment with static perturbation but with higher perturbation amplitude. Figure 9 shows results from a typical discharge in $m / n=12 / 4$ base mode with a perturbation current of $I_{D E D}=13.5 \mathrm{kA}$, i.e. $0.9 \times I_{D E D \max }$ and similar parameters as in the sweep mode discharge discussed before (TEXTOR \#95895 with $I_{p}=365 k A, B_{t}=1.9 \mathrm{~T}, q_{a}=3.6$, $P_{\text {tot }}=600 \mathrm{~kW}, \beta_{\text {pol }} \simeq 0.3, R_{0}=1.73 \mathrm{~m}$ and $\left.a=0.458 \mathrm{~m}\right)$. Again, upper row shows the $n_{e}(r)$ and $T_{e}(r)$ profiles from an unperturbed reference discharge (red profiles) and during DED application (blue profiles). The green line with right ordinate depicts the correspondent $L_{c}(r)$ profile extracted from ATLAS results as convolution with the He beam divergence. The green dashed vertical line depicts the position of the LCFS in the unperturbed discharge. A flattening of the $n_{e}(r)$ profile is obvious while the slope of the $T_{e}(r)$ profile seems to be unaffected. This is emphasized by the corresponding gradient profiles $\nabla n_{e}(r)$ (left image in lower row) and $\nabla T_{e}(r)$ (right image in lower row). The gradient $\nabla n_{e}(r)$ strongly decreases where $L_{c}(r)$ has a dip at around $r=0.425 \mathrm{~m}$. Here the laminar flux tube dominates the observation volume as visualized in supporting figure 8. It acts as SOL opening a domain of parallel transport and particles entering this plasma volume by radial transport are channeled towards the DED target. Further outside $(r=0.44 m) \nabla n_{e}(r)$ increases again approaching the unperturbed level. Here $L_{c}(r)$ increases too, as a remanent island is located in the beam line. In the vicinity of this ergodic subdomain the parallel transport to the wall elements vanishes and the radial particle flux has to be realized by $\nabla n_{e}$ again. This leads to the observed recovery of $\nabla n_{e}$. The dominating parallel particle transport in the laminar flux tube is obviously also the origin for the reduction of $T_{e}$ as nearly no change in $\nabla T_{e}$ can be observed. 
The conductive heat transport remains unchanged while the convective part, rapidly increased due to the laminar flux tube evolving inside the formerly enclosed flux surface region, causes the measured energy loss. This proves the statement that the stochastic edge layer induced by the DED in $m / n=12 / 4$ base mode configuration exhibits an dominant impact of the magnetic perturbation on particle transport. Heat loss from this volume is caused rather by convective transport than by an enhanced radial heat conduction.

This investigation in the fine structured stochastic edge layer in $m / n=12 / 4$ base mode configuration was accompanied by a similar analysis in $m / n=6 / 2$ base mode configuration with extended diagnostic capabilities. The characterization of the stochastic edge layer in both base mode configurations in section 2 showed that for the lower base mode spectrum the typical structural size scale doubles as well as the values of the characteristic ergodisation parameters. The field line diffusion is about twice as high which shall lead to an correspondingly higher enhancement of radial transport.

To demonstrate this effect experimentally, results from two different diagnostics at different poloidal positions are presented here. We start with the analysis of He beam measurements correspondent to the previously presented results. Figure 10 shows $n_{e}(r)$ and $T_{e}(r)$ profiles for two discharges in $m / n=6 / 2$ base mode. They were introduced already for the discussion of the ECE-I data in the beginning. Left column shows results from TEXTOR discharge \#101435 with standard $I_{P}$ and $B_{t}$ direction while in the right column visualizes the correspondent measurement for reversed $I_{P}$ and $B_{t}$ setup (TEXTOR \#101431). The blue profiles depict $n_{e}(r)$ and $T_{e}(r)$ for a reference point without perturbation. The discharges were operated in DED strike point sweep mode and the other two profiles accordingly show the profile change for the two extremal points during sweep. The green profiles were extracted at beginning of the sweep and the red profiles for maximum excursion of the topology. Both points are marked in according colors in the laminar plot shown in the upper row of figure 10. The middle row depicts the selected $n_{e}(r)$ profiles and lower row the selected $T_{e}(r)$ profiles (profiles were extracted averaging over a time window of $50 \mathrm{~ms}$ ).

For standard $I_{P}$ and $B_{t}$ configuration (left column) a laminar flux tube is located in front of the He beam diagnostic and both measurements during sweep were accomplished in this SOL domain of the perturbed edge layer. Accordingly we observe a strong reduction of both $n_{e}(r)$ and $T_{e}(r)$. The profiles are as steep as the unperturbed reference decaying rapidly inside the short $L_{c}$ domain and they are only flattened in the far SOL region at $r=0.45 \mathrm{~m}$. This proves that for this base mode configuration the laminar flux tubes act as SOL also and that they dominate this radial and poloidal area by parallel transport to the wall elements.

This experimentally detected behavior is changed reversing $I_{P}$ and $B_{t}$. The profiles during sweep of the topology become flattened and - as important new finding in this 
base mode $-T_{e}$ is even not reduced for the phase with maximum excursion $\left(\operatorname{red} T_{e}(r)\right.$ profile in right column).

This change in the experimental response onto the DED induced RMP field can be explained analyzing the magnetic topology in this reversed configuration. Upper right figure in figure 10 depicts $L_{c}(r, \vartheta)$ for this discharge and compared to the standard $I_{P}$ and $B_{t}$ settings, now the laminar flux tube is replaced by an ergodic domain. The profiles react accordingly exhibiting a strong flattening of $n_{e}(r)$ and no reduction in $T_{e}(r)$. The parallel transport to the wall elements vanishes in this vicinity and the strong flattening of the $n_{e}(r)$ profile in the ergodic domain $(\mathrm{r}=0.42-0.44 \mathrm{~m})$ points out that here the radial particle transport is much stronger enhanced than in $m / n=12 / 4$ base mode configuration. The gradients $\nabla n_{e}(r)$ decrease by $62 \%$ from $\nabla n_{e}(r=0.43 \mathrm{~m})=1.3 \times 10^{20} \mathrm{~m}^{-3} / \mathrm{m}$ for the unperturbed (blue) profile to $\nabla n_{e}(r=0.43 \mathrm{~m})=0.5 \times 10^{20} \mathrm{~m}^{-3} / \mathrm{m}$ for the perturbed (red) profile in the ergodic region. This means that we detect experimentally an even higher enhancement of radial particle transport than expected by the factor of 2 increase of $D_{f l}$. However, in this base mode not only a strong effect on the particle transport was observed but the heat transport is affected also. Even though particle loss in this volume is observed again, the unchanged $T_{e}$ values in the ergodic domain indicate that here energy loss is less. Examining the green profile which is measured just on the boundary between laminar flux tube and ergodic domain makes clear that here the convective loss affect both profiles much stronger than in the ergodic domain and less compared to the center of the flux tube. This means that in the ergodic domain an enhanced radial heat transport must take place. The enhancement of the radial particle transport discussed before proves this and points out that again the radial particle flux causes convective heat transport. A reduction of $\nabla T_{e}(r)$ in the ergodic domain compared to the unperturbed case was not observed in these measurements and therefore the expected enhancement of radial heat conduction by magnetic braiding can not be confirmed.

To crosscheck and extend this experimental findings the new edge TS system at TEXTOR was used. Figure 11 depicts a set of TS measurements for the same discharges with standard and reversed $I_{P}$ and $B_{t}$ configuration and with perturbation applied $\left(I_{D E D}=3 k A\right)$. This diagnostic is located at top of the TEXTOR vessel at $\vartheta=280 \mathrm{deg}$. The view line is sketched in the laminar plots shown in the upper row of figure 11 and the extracted $L_{c}(r)$ profiles are superimposed as dashed lines with ordinate on right. Red profiles are from the discharge with reversed $I_{P}$ and $B_{t}$ configuration, blue for the standard case.

The same phenomena as discussed before is visible in the magnetic topology for this measurement position also. Reversing $I_{P}$ and $B_{t}$ leads to an exchange of the dominating topological domains in front in the observation volume. This is visible in the $L_{c}(r)$ profiles as in the blue profile an ergodic region with long $L_{c}$ a larger radii persists. The experimental data are affected by this change in the magnetic topology in accordance 
to the discussion of the results from He beam measurement. The SOL flux tube lead to a strong decay of $n_{e}$ and $T_{e}$ while - and this is manifested here in high clarity - the ergodic domain is characterized by a diffusive transport characteristics. Both the $n_{e}(r)$ as well as the $T_{e}(r)$ profile flatten compared to the flux tube dominated measurement. At smaller radii island chains remained and the profiles from both configurations merge. The transport manipulation for this perturbation level is obviously limited to the small edge region where both characteristic domains are interwoven.

These results emphasize the fact the in the DED induced stochastic edge layer two contrary transport domains are existing: the laminar flux tubes acting as helical SOL with a prevailing parallel transport to the wall elements and the ergodic domains with enhanced radial particle transport due to stochastic diffusion of the magnetic field lines. The radial heat transport is only affected by a change in the convective terms due to the increased radial particle transport. An enhanced radial heat conductivity in accordance to classical ergodic transport hypothesis by magnetic braiding was experimentally not detected.

\section{Comparison of experimental results to $3 \mathrm{D}$ modeling}

The findings discussed before on experimental basis were subsequently investigated by three dimensional (3D) transport modeling with the EMC3/EIRENE code. This code solves a set of stationary Braginskii fluid equations (EMC3) for plasma density, parallel momentum and temperature of electrons and ions with a Monte Carlo (MC) method. It is coupled to the 3D kinetic Monte Carlo code EIRENE [34]. Here the source terms for particle, energy and momentum from recycling neutrals are calculated in a kinetic treatment by an iterative input-output calculation with EMC3. For a more detailed description of the code please see $[17,35]$ and references therein. This numerical investigation focused in particular on the question if the experimentally detected poloidal asymmetry and their explanation by the magnetic topology, i.e. the two characteristic transport domains, can be reproduced. In addition the 3 -D code allows to extend the experimental information from specific diagnostic locations and spatial observation areas along the whole toroidal angle. Therefore we aimed at a three dimensional resolution of the magnetic topology and the correlated plasma structures. However, as this was the initial attempt to perform direct comparison to experiment at TEXTOR-DED the first step was to benchmark the code and check for fundamental consistency. In this section results from these three major priorities for this initial modeling are presented.

The code incorporates estimates on the anomalous radial transport into the Monte Carlo step length. Therefore the perpendicular particle diffusion $D_{\perp}$ and heat diffusion coefficient $\chi_{\perp}$ are needed as input from experiment. As experimental basis for this first comparative modeling discharge \#95985 $\left(I_{D E D}=13.5 \mathrm{kA}\right)$ in $\mathrm{m} / \mathrm{n}=12 / 4$ base mode configuration and the unperturbed reference discharge \#95896 were used. 
The experimental results of this discharge were analyzed on the basis of the He beam measurements as described in beginning of section 3. These measurements cover the important part of the stochastic edge in this base mode and they are therefore an ideal input for modeling. The transport coefficients were deduced from the unperturbed reference discharge \#95896 on the basis of the simple SOL model of Stangeby [36] from the experimental $n_{e}(r)$ and $T_{e}(r)$ profiles and their e-folding lengths $\lambda_{n}=21 \mathrm{~mm}$ and $\lambda_{T}=50 \mathrm{~mm}$ to $D_{\perp}=1.4 \mathrm{~m}^{2} \mathrm{~s}^{-1}$ and $\chi_{\perp}=4.1 \mathrm{~m}^{2} \mathrm{~s}^{-1}$. Together with the values $n_{\text {ebnd }}(r=0.375 \mathrm{~m})=1.2 \times 10^{19} \mathrm{~m}^{-3}$ (deduced from HCN interferometer measurements) at the inner boundary and the heating power $P_{H}=600 \mathrm{~kW}$ these parameters are the boundary conditions for modeling.

The upper plot in figure 12 shows the radial and poloidal distributions $n_{e}(\vartheta, r)$ and $T_{e}(\vartheta, r)$ for full poloidal circumference. It is clearly visible that the poloidally asymmetric topology in the stochastic edge region shown in figure 2 and in particular in figure 3 (left plot) is reflected in the modeled plasma structure. The Poincaré plot overlaid shows that a reduction of both parameters is observed where the magnetic field gets a stochastic structure with interleaved ergodic and short $L_{c}$ regions. In close correspondence to this topology the $n_{e}$ and $T_{e}$ values are modulated. A stronger reduction takes place where the short $L_{c}$ flux tubes are located. In the ergodic region $n_{e}$ and $T_{e}$ are higher.

For a more detailed comparison of plasma structure and magnetic topology poloidal profiles of $n_{e}(\vartheta)$ and $T_{e}(\vartheta)$ and of the connection length $L_{c}(\vartheta)$ at $r=0.43 m$ were extracted and compared. We choose a grid cell index in the modeling grid which is representing an unperturbed flux surface at $r=0.43 \mathrm{~m}$ in the reference discharge. The direct comparison of both, $n_{e}(\vartheta)$ and $T_{e}(\vartheta)$ with $L_{c}(\vartheta)$ is shown in the lower plots of figure 12. The poloidal modulation of both electron parameters becomes obvious and the correlation with the analog modulation of $L_{c}(\vartheta)$ confirms the experimental finding on a strong poloidal asymmetry of the perturbed edge layer. Modeling also shows that the short $L_{c}$ flux tubes cause a strong reduction of $n_{e}(\vartheta)$ and $T_{e}(\vartheta)$ strengthening the experimental finding that here a channel of direct parallel transport towards the DED target is opened. This is in particular reliable as the code's equations do not include an self consistent electrical field or potential changes as quasi neutrality is assumed. The particle and energy loss in the laminar flux tube observed in modeling therefore must be driven by parallel particle flows towards the target as in a classical SOL description. The origin of this flows are the parallel density and temperature gradients between the upstream position at LFS and the strike point region on the DED target.

In order to evaluate in how far this mechanism is described quantitatively correct by the code the reduction of $n_{e}$ nd $T_{e}$ inside of the SOL flux tubes at LFS in experiment and modeling was deduced and compared. The radial particle and energy fluxes in the ergodic domain into the short $L_{c}$ flux tubes are determined by radial transport in 
the ergodic volume and by the parallel fluxes inside of the SOL flux tubes themselves. Therefore the reduction of $\hat{n}_{e}$ and $\hat{T}_{e}$ (values normalized to the unperturbed reference discharge) is a proper measurement for the SOL functionality of the laminar flux tubes. In experiment a drop to 0.5 for $\hat{n}_{e}$ and 0.7 for $\hat{T}_{e}$ was determined in comparison to the reference discharge. In contrast a reduction of both parameters to $\hat{n}_{e}=0.5$ and $\hat{T}_{e}=0.55$ was deduced from modeling. This means that here the heat transport is overestimated.

To investigate the impact of the background transport parametrised in $D_{\perp}$ and $\chi_{\perp}$ on this finding we decreased these values subsequently as follows: $D_{\perp}=1.4 \mathrm{~m}^{2} \mathrm{~s}^{-1} \rightarrow$ $0.5 \mathrm{~m}^{2} \mathrm{~s}^{-1}$ and $\chi_{\perp}=4.1 \mathrm{~m}^{2} \mathrm{~s}^{-1} \rightarrow 3.0 \mathrm{~m}^{2} \mathrm{~s}^{-1}$. This reduction of radial transport is motivated by the idea that with decreased radial fluxes the impact of the parallel transport towards the target must become relatively larger. And indeed we elaborated that a higher reduction to $\hat{n}_{e}=0.45$ and $\hat{T}_{e}=0.5$ takes place after this manipulation of $D_{\perp}$ and $\chi_{\perp}$. However, this means that $T_{e}$ is even more reduced and that $\hat{T}_{e}$ is diverging from the experimental finding. This first input parameter modulation suggests that the heat transport in the flux tubes is not yet properly reflected in the code equations. One ongoing approach for correction is to include a flux limit for the parallel heat flux [35].

Concerning the aim to extend the spatial information of localized measurements with modeling results the experimental findings were confirmed and completed in two more important points. First the comparison of the magnetic topology in figure 2 and figure 3 (left figure) with the modeling results show that the short $L_{c}$ flux tubes are elongated for poloidal angles in the vicinity of the DED target region [17]. In front of the target - and this is the second interesting fact - finger like structures are formed into which both domains are embedded, the elongated flux tubes as well as the ergodic supplements around them. These fingers are separated by regions with very short $L_{c} \approx 5 m$ ( $\hat{=} 0$ p.t.) and without a direct magnetic connection neither into the laminar nor into the ergodic domains. Therefore this region is expected to be separated from the plasma streaming inside the fingers onto the DED target and it is termed as the private flux region (PFR) of the DED induced ergodic divertor structure. This numerical finding was confirmed by measurements performed with the He beam diagnostic right in front of the DED target. They showed that the pressure between the finger structures sharply drops down to about $20 \%$ of the unperturbed reference value proving vanishing particle and heat fluxes into this subdomain [32]. The finger structure separated by the PFRs obviously determines the plasma structure in front of the DED target as confirmed by several experimental analysis described in references [15], [28] and [27] for example.

In order to elaborate the impact of decreasing base mode numbers on these findings a modeling based on the experimental data from discharge \#95896 $\left(D_{\perp}=\right.$ $1.4 \mathrm{~m}^{2} \mathrm{~s}^{-1}, \chi_{\perp}=4.1 \mathrm{~m}^{2} \mathrm{~s}^{-1}$ and same boundary conditions as before) was performed with $I_{D E D}=6.75 \mathrm{kA}$ in $\mathrm{m} / n=6 / 2$ base mode configuration. Figure 13 depicts the results in in same systematics as figure 12 for $m / n=12 / 4$ base mode. The extension 
of the typical structural sizes discussed on the experimental basis in figure 3 is here clearly visible in the modeled plasma structure. In poloidal direction the mode number is decreased with an according increase of the poloidal $n_{e}(\vartheta)$ and $T_{e}(\vartheta)$ modulation wavelength. In radial direction we clearly can see that in contrast to the extension of the SOL flux tubes in $m / n=12 / 4$ base mode, here the radial domain with modulating $n_{e}$ and $T_{e}$ values is extended. In $m / n=12 / 4$ base mode this manipulated region was limited to a region with $\Delta r \approx 4 \mathrm{~cm}$ width while in $m / n=6 / 2$ base mode the radial width is increased to $\Delta r \approx 8 \mathrm{~cm}$. This was expected from magnetic modeling and confirmed by the experimental results and the 2D cuts from 3D modeling shown here.

As physics of neutral particles is incorporated into the EMC3/EIRENE modeling by the EIRENE code, the momentum equations can be solved [35] allowing calculations of the plasma flow pattern $M_{N}(r, \vartheta)$. In experiment access to these quantity is not given. However, as discussed before in particular parallel particle flows to the DED target are suggested as major player for the functionality of the DED induced stochastic edge layer. So far only the modeling can give us correlated information.

Figure 14 shows the two dimensional distribution of the mach number $M_{N}(r, \vartheta)$. Here several distinct plasma flow regions appear. First a SOL flow in the radial vicinity of the limiter tiles, i.e. on the DED target sides and on the ALT-II limiter is visible. Second, in the stochastic edge layer the poloidally modulating connection length distribution $L_{c}(r, \vartheta)$ strongly affects the $M_{N}(r, \vartheta)$ pattern. Twelve - this means $m \times 2$ - neighboring and counter streaming flux tubes evolve which have a SOL characteristic, i.e. $M_{N}$ increases in front of the target to unity satisfying the Bohm criterion. This confirms that in the laminar flux tubes plasma flows are generated by parallel gradients which depend on the length of the magnetic field line.

To understand the $M_{N}$ pattern exactly we have to consider the relation to topology in more detail. Hereto we discuss the poloidal profiles $M_{N}(\vartheta)$ with the connection length profile. This comparison is shown in figure 15 at radial position $r=0.41 \mathrm{~m}$. In the upper plot $M_{N}(\vartheta)$ is compared to $L_{c}(\vartheta)$ while in the lower plot another kind of field line connection length is elaborated. As in this paper the connection length $L_{c}$ is consistently defined as length from target to target this definition means that from a given poloidal plane the shortest connection towards the DED target can be different from $L_{c}$ and it has different values in positive and negative toroidal direction. In a linear density regime $M_{N}$ will be higher on the shorter pathway as we are closer to the target in a given flux tube. On the longer pathway to the target $M_{N}$ will be less with correlated impact on density. The information needed for this analysis is the so called shortest connection length which is compared to $M_{N}(\vartheta)$ in the lower part of figure 15. Here it becomes obvious that parts of the long $L_{c}$ domain existent in the upper plot are vanishing evaluating the shortest pathway to the targets. In the quantity of short connection length only a region with poloidally very small extension persists where the field lines in both direc- 
tion have long $L_{c}$. However, this means that the formerly long field lines have a long connection into one and a short connection into the other direction. The profile $M_{N}(\vartheta)$ is modulated accordingly, i.e. it exhibits a reversal in slope were the small peak with long field lines exists. Channels on the left and on the right of these long $L_{c}$ peaks connect into different toroidal directions to the DED target. Therefore $M_{N}$ reverses its sign.

After we now understand how the flow pattern is governed by the topology we try to resolve the correlation to the $n_{e}$ and $T_{e}$ reaction as discussed before. Figure 16 depicts a comparison of poloidal profiles of $n_{e}(\vartheta)$ (upper plot) and $T_{e}(\vartheta)$ (lower plot) with $M_{N}(\vartheta)$ at $r=0.41 \mathrm{~m}$. This is the radial position corresponding to the poloidal profiles shown in figure 13 already and this comparison strengthens our conclusions on the role of parallel flows for transport in the stochastic edge layer. In the vicinity where $M_{N}$ has positive values $n_{e}$ is reduced. Here field lines connect in both directions directly to the target as visualized in the upper plot of figure 15. These field lines form the laminar flux tubes, they are radially and poloidally trapped in helical magnetic flux tubes and they do not connect to deeper radial regions. On the other hand where $L_{c}$ is long, the $M_{N}$ values are negative as field lines here connect along the short path toroidaly in the other direction. Along the long path these field lines diffuse from radially deeper regions into this domain transporting particles and energy via the flow quantified by the $M_{N}$ value. This shows that the magnetic diffusion enhances radial transport as the parallel transport along field lines is projected into the radial direction. As concluded before the parallel flow along magnetic field lines transports particles and energy in the regions classified as ergodic leading to increased $n_{e}$ and $T_{e}$ values here. This confirms the explanation of the modulated $n_{e}(\vartheta)$ and $T_{e}(\vartheta)$ profiles.

However, comparing the correlation of $n_{e}(\vartheta)$ with $M_{N}(\vartheta)$ on the one and of $T_{e}(\vartheta)$ with $M_{N}(\vartheta)$ on the other hand there is a clear difference visible. The $n_{e}(\vartheta)$ profile is closely correlated to the $M_{N}(\vartheta)$ profile while for $T_{e}(\vartheta)$ another mechanism seems to be existent as $T_{e}$ is not always reduced when significant flows appear (e.g. between $\vartheta=310-330 \mathrm{deg}$ in figure 16). This observation is explained taking again $L_{c}$ into account. We can see in figure 15 that the poloidal region $\vartheta=310-330 \mathrm{deg}$ exemplarily chosen here is ergodic in our classification as $L_{c}$ is high and we know that field lines from deeper plasma regions connect to this domain. This means that we have a large fraction of parallel heat conduction beyond the convective level specified with $M_{N}$. As soon as we enter a domain of positive $M_{N}$, i.e. a laminar flux tube, $T_{e}$ is largely reduced also by the direct particle loss to the wall elements.

This consistent findings support the analysis of the radial heat and particle transport characteristics. In figure 17 the radial $n_{e}(r)$ and $T_{e}(r)$ profiles are shown at two different poloidal position. Left figures show these profiles in the vicinity of an ergodic domain where a large fraction of long $L_{c}$ field lines influences transport. Flat $n_{e}$ and $T_{e}$ profiles are visible in this vicinity at $r=0.38-0.41 \mathrm{~m}$. In contrast the opening of a laminar 
flux tube shown in plots on the right exhibits the SOL functionality. Steep gradients at the inner edge can be seen and a reversal of $\nabla n$ inside of the laminar flux tube. The persistent long $L_{c}$ domain further outside is filled by parallel transport from deeper plasma regions leading to even increased $n_{e}$ and $T_{e}$ values. These observations are well correlated to the experimental findings discussed on basis of figure 10 and 11.

In summary these findings from modeling confirm systematically the basic mechanisms for particle and heat exhaust in the stochastic edge of TEXTOR-DED from the previous experimental analysis: the laminar flux tubes as helical SOL domains channel the radial influx from the ergodic domains towards the DED target. Here convection is the major channel for heat transport while along the long field lines in the ergodic domain less convective losses appear. Here stronger parallel conduction leads to radial heat transport in the ergodic regions adjacent to the SOL flux tubes. So far we used the code as tool to confirm the experimental findings and enhance the meaning of the analysis by new information on the $M_{N}$ pattern from modeling. However, as these are results from first modeling attempts in direct comparison to experiment we now describe results from code validation and discuss the consequences for improvement and further development. First results from these suggestions are described in [35, 32].

The EMC3/EIRENE code package is applicable also for three dimensional modeling of particle and energy deposition on the DED target [17]. This allows a very direct comparison of the code results with experimental data. Therefore we used the deposition pattern on DED target for further code validation. On the experimental side data from two camera systems were used. First a $H_{\alpha}(\lambda=656.5 \mathrm{~nm}, \Delta \lambda=15 \mathrm{~nm})$ filtered CCD camera was applied. This system has a line of sight perpendicular to the DED target. Hereby this camera integrates the $H_{\alpha}$ emission along the line of sight and therefore the light intensity was used as a measure for the particle flux density $\Gamma_{\perp}(\vartheta, \varphi)$ onto the DED target. Second, an infrared camera system was applied to measure heat flux density $q_{\perp}(\vartheta, \varphi)[31]$. Figure 18 shows a comparison of modeled and measured deposition pattern. In the upper row $\Gamma_{\perp}(\vartheta, \varphi)$ from modeling (left figure) and from experiment (right figure) is shown. Lower row shows $q_{\perp}(\vartheta, \varphi)$ in the same ordering, modeling result on the left and experimental result on the right. These results visualize that the magnetic perturbation induced by the DED forms a helical, multipolar divertor structure. The thin finger structure discussed before channels the particle load into this helical deposition pattern which consists for $m / n=12 / 4$ base mode out of 4 stripes split into two separate strike zones separated by PFRs. In $m / n=6 / 2$ base mode the number of base stripes reduces to two in accordance to the toroidal mode number. For high perturbation amplitude these stripes again split into two separate stripes .

Comparing the deposition pattern from modeling and experiment in figure 18 this helical structure is exhibited in the experimental pattern also. The splitting of the target pattern for this level of perturbation is visible and the helicity and inclination of the 
pattern appears to be the same. This proves that the code incorporates the divertor functionality of the DED induced stochastic boundary, i.e. the SOL functionality of the helical, laminar flux tubes depositing the channeled particle and energy fluxes into the helical target strike point pattern. To enhance this qualitative finding on the correspondence between modeling and experiment poloidal profiles $\Gamma_{\perp}(\vartheta)$ and $q_{\perp}(\vartheta)$ at $\vartheta=170 \mathrm{deg}$ in experiment were extracted and compared to the corresponding profiles from modeling (position shown as dashed line in left plots of figure 18). The results are depicted in figure 19. Plot on the left shows $q_{\perp}(\vartheta)$ from modeling (red profile with left ordinate) and from experiment (dashed green profile, right ordinate, $T_{\text {surf }}$ is shown as measure for $q_{\perp}$ ), while plot on the right shows $\Gamma_{\perp}(\vartheta)$ with result from modeling as blue profile with ordinate on the left and data from experiment as green profile with ordinate on the right. For $\Gamma_{\perp}$ a very good agreement between modeling and experiment is found. This ensures on the first level that the implementation of the hardware geometry and of the magnetic equilibrium into the code is consistent with the real experimental setup. Beyond this basic crosscheck this result supports the comparison of the deposition pattern and shows in detail that particles and energy are deposited in the separates stripes of the strike zones. They are discriminated by the PFRs with very short $L_{c}$. Here $p_{e}$ sharply drops and in accordance the fluxes also. However, the experimentally obtained heat distribution on the target does not reflect the exact modeled structure. The position of the main maxima coincides but the width of the peak and the substructures of the stripes are not reflected. This is a problem of the experimental setup as there is a small misalignment of the DED target tiles which can practically not be implemented into the code. As heat deposition is very sensitive to these missalignement the chance to resolve fine structures depends very much on the target shape.

As final step for this initial numerical analysis of transport and code validation we compared the experimental $n_{e}(r)$ and $T_{e}(r)$ profiles to the profiles from modeling with both sets of transport coefficients. For this task we extracted the modeled profiles at $\vartheta=5 \mathrm{deg}$, the location of the He beam diagnostic. In addition to $n_{e}(r)$ and $T_{e}(r)$ deduced with this diagnostic technique, we used results for $n_{e}(r)$ from a thermal lithium beam diagnostic at the same location [11]. The results of this comparison are shown in figure 20. The upper row shows the profiles for the reference discharge. The left plot depicts $n_{e}(r)$ from experiment (blue profile from He beam, red profile from Li beam) and from modeling. The color code of the modeled profiles marks the profiles from modeling with $D_{\perp}=1.4 \mathrm{~m}^{2} \mathrm{~s}^{-1}$ and $\chi_{\perp}=4.1 \mathrm{~m}^{2} \mathrm{~s}^{-1}$ (experimentally deduced values $\rightarrow$ modeling 1) in green and the results from modeling with decreased values (modeling 2) as black profiles. In the lower row the same combination of profiles is depicted for the perturbed discharge.

Based on the unperturbed reference discharge we can judge if the settings of the boundary conditions lead to appropriate correspondence between the experimental and the modeled profiles. For both, $n_{e}(r)$ as well as $T_{e}(r)$ there is a fair match between the 
green profiles from modeling 1 and experiment. Especially at larger radii $(r>0.45 \mathrm{~m})$ $n_{e}(r)$ from the Li beam measurement matches the green modeled profile. Here the boundary conditions define the decay of the profile and a good correspondence was expected. However, as the values of $D_{\perp}=1.4 \mathrm{~m}^{2} \mathrm{~s}^{-1}$ and $\chi_{\perp}=4.1 \mathrm{~m}^{2} \mathrm{~s}^{-1}$ are significantly high, this good correspondence is a satisfying proof of a solid deduction of the boundary values by the simple SOL model of Stangeby. This is especially pronounced in the good match of $T_{e}(r)$ profiles from modeling 1 and experiment. Decreasing $\chi_{\perp}$ leads to a lower radial energy flux in this volume and therefore $T_{e}(r)$ rises with a significant deviation from experiment. In contrast the $n_{e}(r)$ profile from He beam measurement in the inner experimental observation volume is in better correspondence to the profile from modeling 2 with reduced $D_{\perp}$. This suggests that the radial transport in the discussed observation volume may change and therefore the input for anomalous radial transport should be a function of radius $D_{\perp}(r)$ and $\chi_{\perp}(r)$ rather than one fixed value in the whole radial modeling domain.

Elaborating this profile comparison for the perturbed discharge, for $n_{e}(r)$ a similar conclusion can be drawn. A reduction of $n_{e}$ in modeling and experiment is clearly visible as discussed before. The Li beam profile at larger radii again matches the profile from modeling 1 while at smaller radii the profile with reduced $D_{\perp}$ describes the experimental result in better correspondence. For $T_{e}(r)$ from modeling 2 the whole profile is manipulated much stronger by the RMP field than the experimental one. A flattening can be observed in the whole radial domain while in experiment the profile shape is only marginally influenced but a reduction of $T_{e}(r)$ along the whole radial profile was observed. This contrary result from experiment and modeling was discussed already before based on the normalized $\hat{n}_{e}$ and $\hat{T}_{e}$ values inside of the laminar flux tubes. In the profile comparison performed here the reason for this deviation becomes obvious. At small radii $T_{e}(r)$ from modeling 2 matches the experimental profile well while at larger radii $\chi_{\perp}$ from modeling 1 seems to be a better candidate as here the modeled profiles are in better agreement with the experimental ones. This supports the idea resumed already during the discussion of the profiles from reference discharge, that the input parameters $D_{\perp}$ and $\chi_{\perp}$ should be a function of the radial coordinate. In addition we already concluded that a proper implementation of flux limits for the conductive energy transport is mandatory.

These results from an initial quantitative code validation support on the one hand the findings on the characteristic transport mechanisms, i.e. the existence of laminar and ergodic domains. On the other hand they give rise to code improvement with special respect on application in plasmas with transport barriers, where a radial dependence of the anomalous transport is of fundamental importance. 


\section{Conclusion and Outlook}

The experimental and numerical results presented in this paper resolved the basic transport characteristics of the DED induced stochastic edge layer in $m / n=12 / 4$ and $m / n=6 / 2$ base mode configuration. Two poloidally adjacent transport domains were identified: a domain representing a helical scrape off layer (SOL) is formed by field lines with short connection and therefore prevailing parallel transport to the wall elements. Here, the field lines are clustered into extended flux tubes embedded into a long connection length ergodic domain with diffusive transport characteristics and enhanced radial transport. This transport manipulation is driven by an enhancement of the radial particle transport leading to an increase of the convective terms in heat transport. An enhancement of radial heat conduction by magnetic braiding was not detected in experiment.

The structural extension of these characteristic domains depend on the base mode configuration and an according extension of the stochastic edge layer in radial direction and the typical structure sizes in poloidal direction were resolved experimentally. The base mode number is reflected in the poloidally alternating plasma structures which are caused by the ergodic and the laminar transport domains. This shows that analysis approaches in the frame of ergodic transport theories can be applied only in the ergodic domains while in the SOL flux tubes a simple SOL approach already leads to sufficient transport estimates for EMC3/EIRENE modeling. Based on these findings the code was validated against the experimental results. Here a satisfying correspondence to the experimental results was shown. However, in detail quantification of the profile reaction in experiment and modeling suggested code improvement, i.e. the implementation of flux limits for parallel conductive heat flow as well as the implementation of a radial function in $D_{\perp}(r)$ and $\chi_{\perp}(r)$.

These new findings encourage us to present the DED as a flexible facility to investigate in detail important aspects of transport and structural formation in stochastic edge layers. The capability to change the base mode configuration on a week to week basis allows to change the typical geometric scales in the magnetic topology and by this to investigate the impact of the base mode spectrum on the performance for edge transport control. The numerical tools to model the magnetic topology (ATLAS and GOURDON) and to analyze the transport behavior in three dimensional edge plasmas (EMC3/EIRENE) in combination with an advanced edge diagnostic equipment makes a thorough code validation possible. These codes are then the tool to relate the basic results obtained at TEXTOR-DED to the promising H-mode discharges with ELM suppression by RMP fields at DIII-D and $J$ ET as well as to results on divertor physics in stellarators or helical devices as $W 7-X$ and $L H D$. The investigations presented here contribute to the assessment of the potential to control heat and particle exhaust in future fusion devices by resonant magnetic perturbations. 


\section{Acknowledgements}

This work was partially supported by the research grant Sonderforschungsbereich (SFB) 591 of the German research fund Deutsche Forschungsgemeinschaft and the European task force on plasma wall interaction.

\section{Figures}

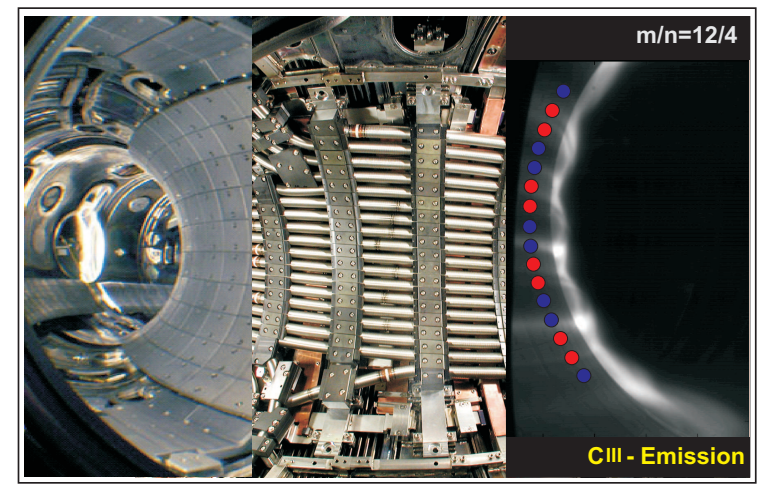

Figure 1: Setup of the DED coils (middle), tangential view into the vacuum vessel with DED target (left) and impression of the DED induced edge structure in CIII light (right, with DED coils, blue $=+11 k A$, red $=-11 k A$ ). 


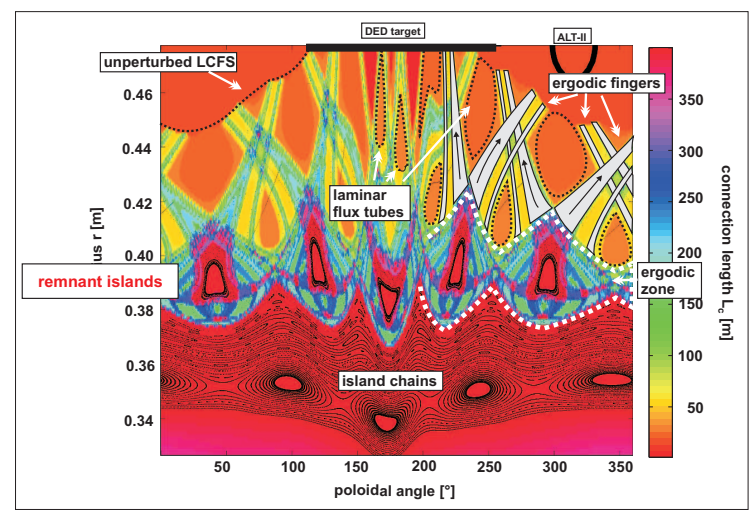

Figure 2: Overview of the magnetic topology in the stochastic edge for $m / n=$ $6 / 2$ base mode configuration. Shown here is a superposition of a Poincaré plot (black colored intersection points with the chosen poloidal plane), a laminar plot (color coded connection length distribution) and highlighted are the characteristic topological domains.
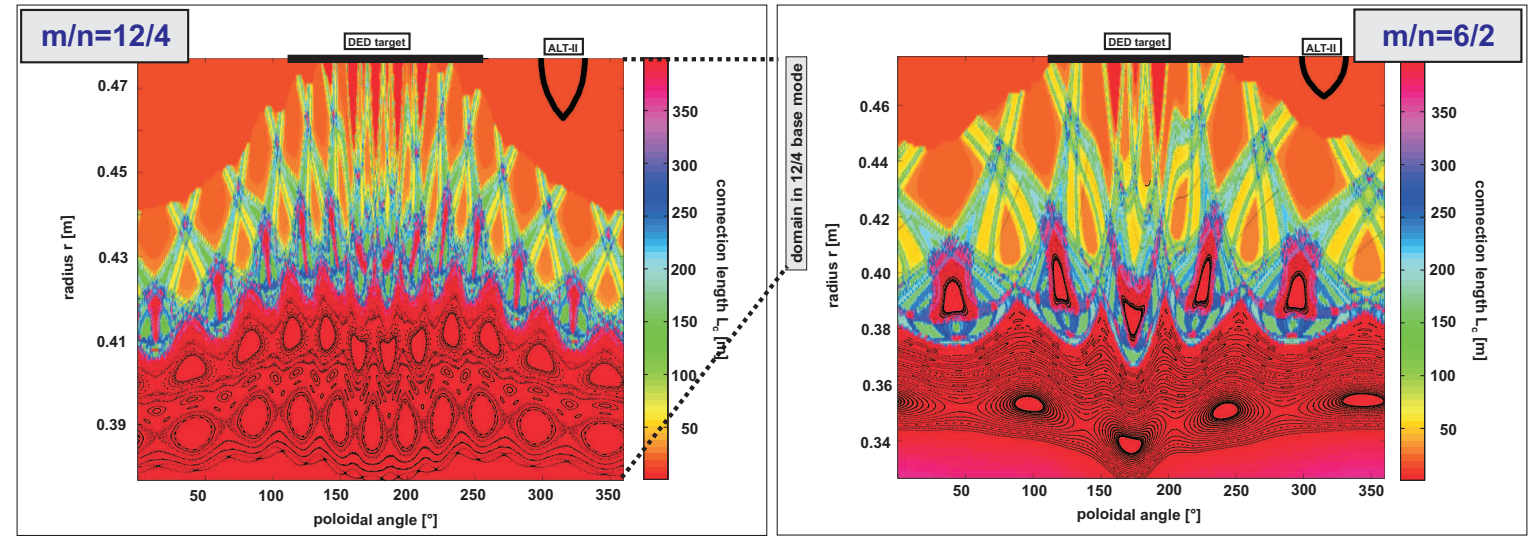

Figure 3: Comparison of magnetic topologies for $m / n=6 / 2$ and $m / n=12 / 4$ base mode configuration. The extension of the typical size of the characteristic domains with decreasing base mode number is visualized. 


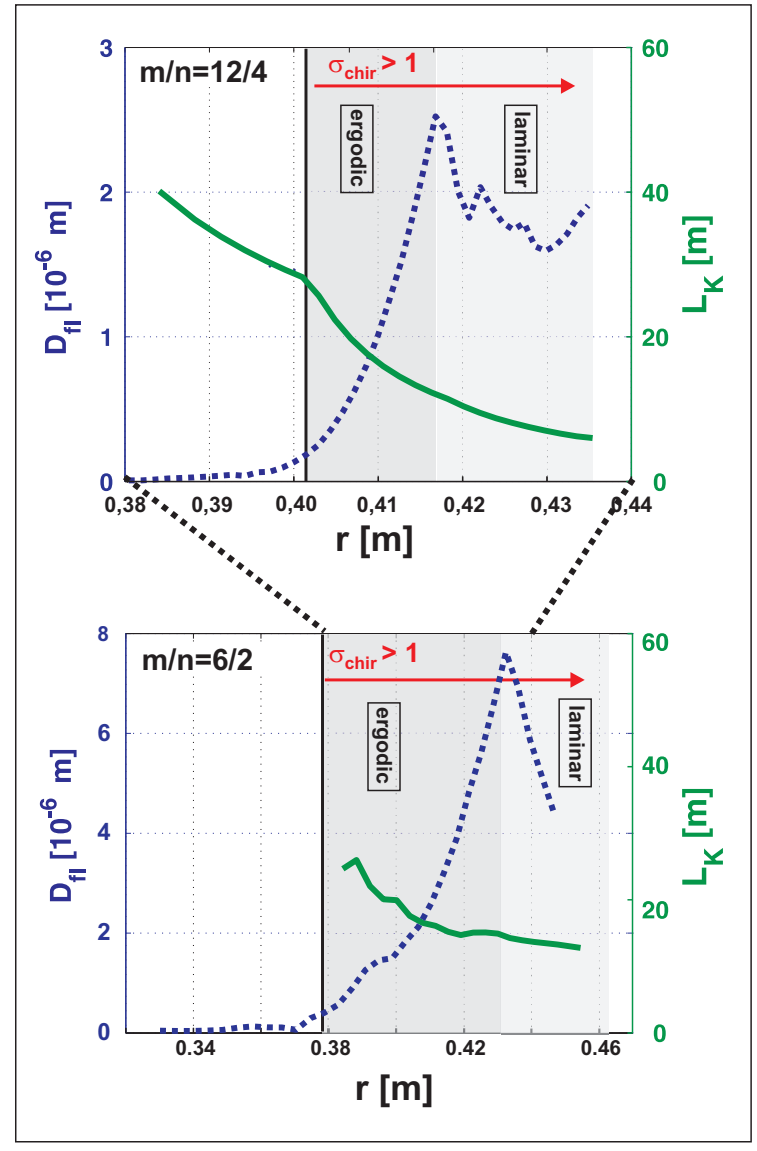

Figure 4: Comparison of radial profiles for characteristic stochasticity parameters commonly used to estimate the impact of stochasticity on transport: Chirikov parameter $\sigma_{\text {Chir }}$ (area where $\geq 1$ is marked with red arrow), field line diffusion coefficient $D_{f l}(r)$ (blue, dashed curve, left abscissa) and Kolmogorov Length $L_{K}(r)$ (green, solid curve, right abscissa). Upper plot for $m / n=12 / 4$ base mode and lower plot for $m / n=6 / 2$ base mode, please note that radial coordinate is zoomed for better resolution. 


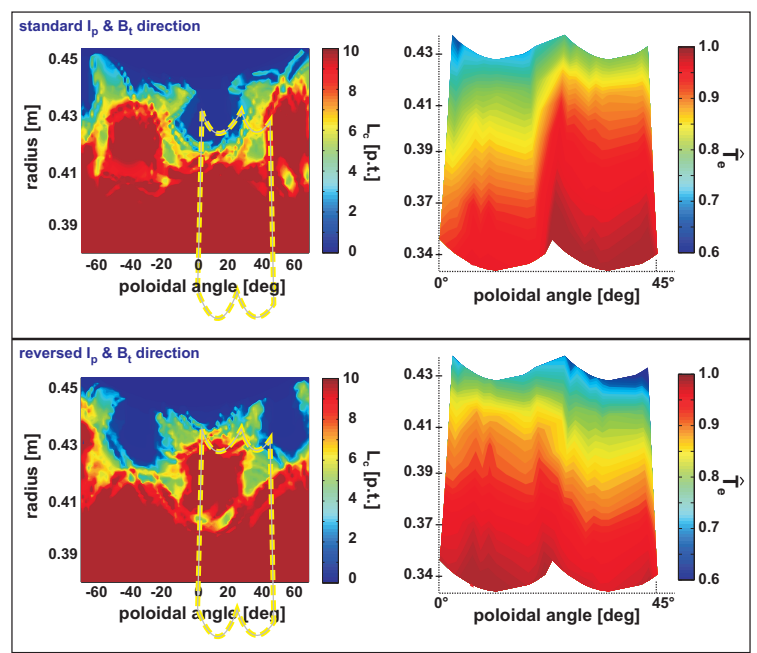

Figure 5: Two dimensional normalized $T_{e}(r, \vartheta)$ distribution (right plots) measured with the ECE-I system in $m / n=$ $6 / 2$ base mode. The results shown are obtained in standard (upper plots, TEXTOR \#101435) and reversed (lower plots, TEXTOR \#101431) $I_{P}$ and $B_{t}$ settings. Left plots show the correspondent $L_{c}(r, \vartheta)$ distribution calculated with the ATLAS code. 


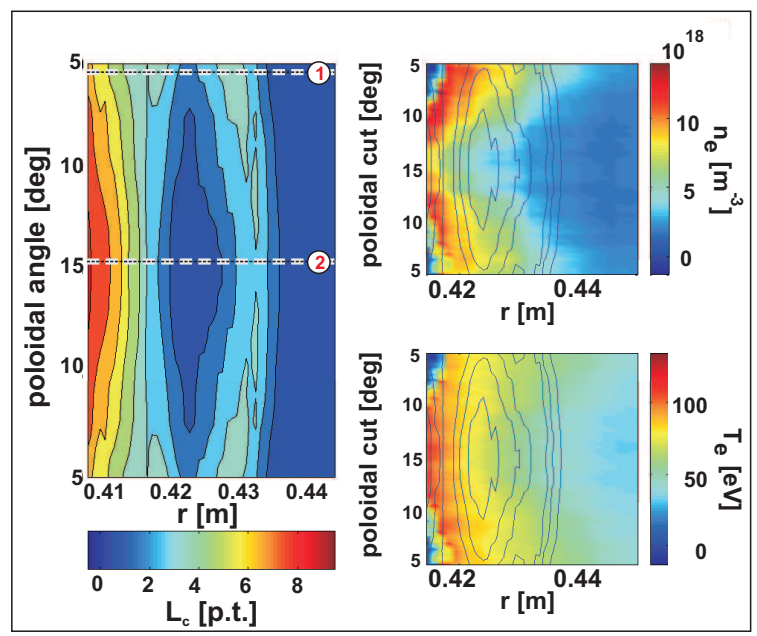

Figure 6: Two dimensional $n_{e}(r, \vartheta)$ and $T_{e}(r, \vartheta)$ distribution (right plots) measured with the He beam diagnostic at LFS during a strike point sweep in $m / n=$ 12/4 base mode (TEXTOR \#95924). The plot on left shows the correspondent $L_{c}(r, \vartheta)$ distribution as convolution of ATLAS output with the beam divergence in poloidal and toroidal direction. Dashed lines mark the extremal points of the sweep: $1 \rightarrow$ start of sweep in vicinity of long $L_{c}$, i.e. ergodic domain and $2 \rightarrow$ maximal excursion by sweep, i.e. laminar flux tube with short $L_{c}$. 


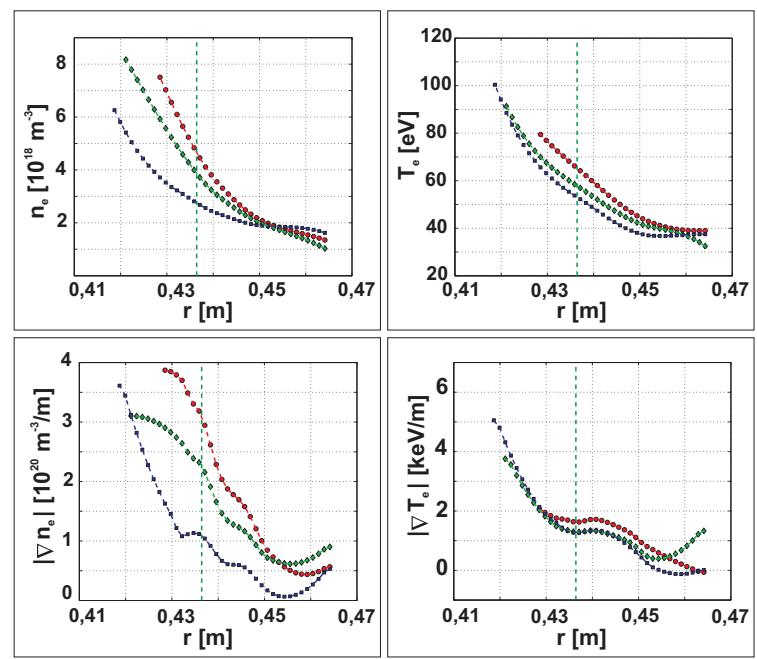

Figure 7: $n_{e}(r)$ and $T_{e}(r)$ extracted from He beam measurement at LFS during strike point sweeping (TEXTOR \#95924, $m / n=12 / 4$ base mode, $\left.I_{D E D}=7.5 k A\right)$ and gradient profiles $\nabla n_{e}(r)$ and $\nabla T_{e}(r)$. Profiles are extracted for the marked stages during the discharge: red o markers $\rightarrow$ phase without perturbation field, green $\diamond$ markers $\rightarrow$ phase in vicinity of long $L_{c}$ (ergodic domain, line 1 in figure 6), blue squared markers $\rightarrow$ phase in vicinity of short $L_{c}$ (laminar flux tube, line 2 in figure $6)$.

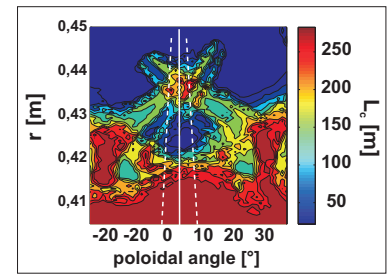

Figure 8: $L_{c}(r, \vartheta)$ at He beam location (depicted as white lines) for $\# 95895, I_{D E D}=$ $13.5 \mathrm{kA}, \mathrm{m} / \mathrm{n}=12 / 4$. 


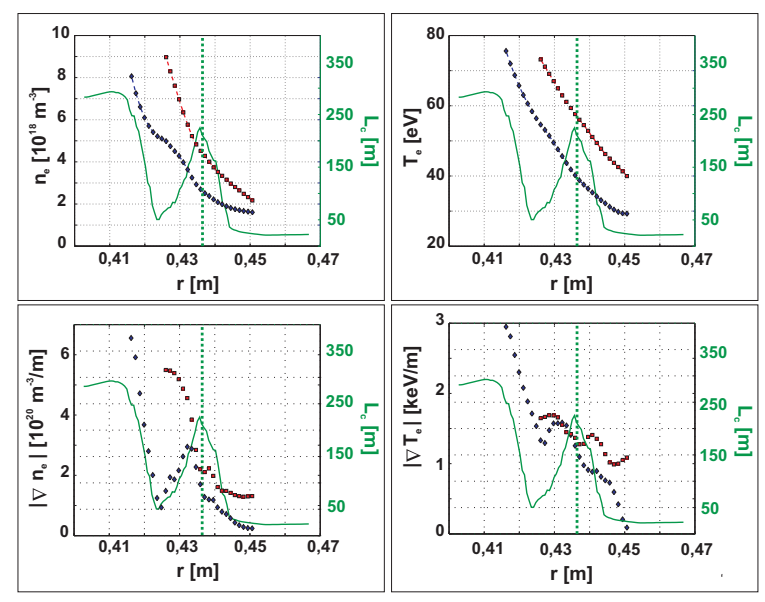

Figure 9: $n_{e}(r)$ and $T_{e}(r)$ extracted from He beam measurement at LFS and gradient profiles $\nabla n_{e}(r)$ and $\nabla T_{e}(r)$. Measurements were obtained for high perturbation case (TEXTOR \#95895, $m / n=12 / 4$ base mode, $I_{D E D}=13.5 \mathrm{kA}$, blue $\diamond$ markers, left abscissa) and are compared to a reference with out perturbation (TEXTOR \#95896, red squared markers, left abscissa). Green solid curve shows the correlated $L_{c}(r)$ profile for \#95895 (see figure 8) with abscissa on right and the dashed green line represents the LCFS with out perturbation field applied. 


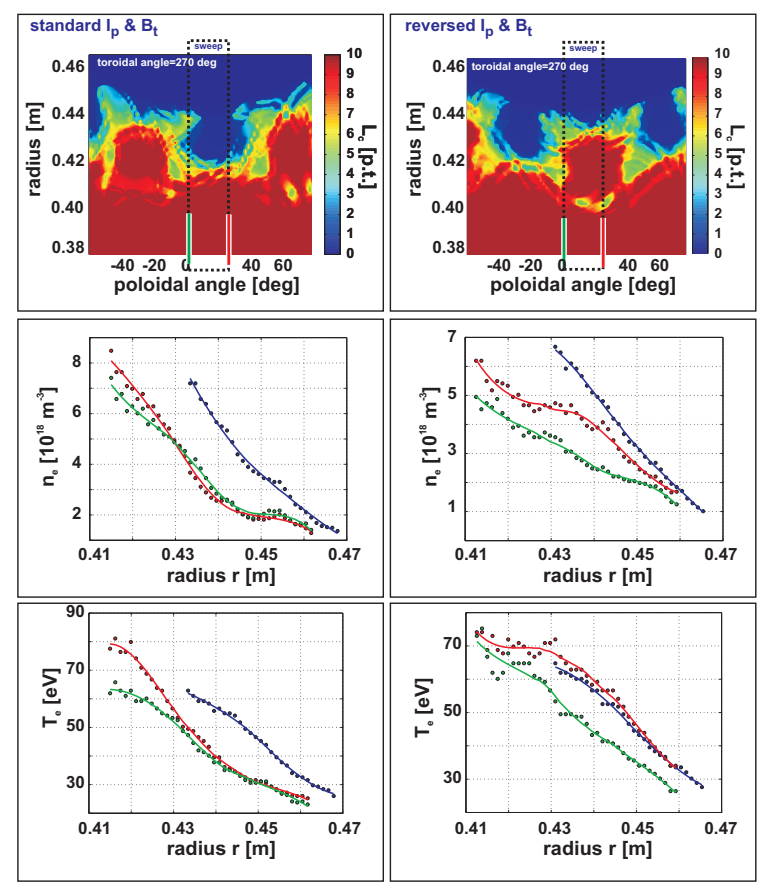

Figure 10: Profile changes in in $m / n=$ 6/2 base mode configuration at LFS: upper row shows the $L_{c}(r, \vartheta)$ calculation during strike point sweeping for standard $I_{P}$ and $B_{t}$ setting (\#101435, left column) and reversed $I_{P}$ and $B_{t}$ setting (\#101431, right column). In middle row $n_{e}(r)$ and in lower row $T_{e}(r)$ are shown extracted from He beam measurement at LFS during strike point sweeping. The profiles were extracted again for the phase with out DED (blue o marker) and during DED strike point sweep on start of the sweep (green $\circ$ marker) and for full excursion (red $\circ$ marker). 


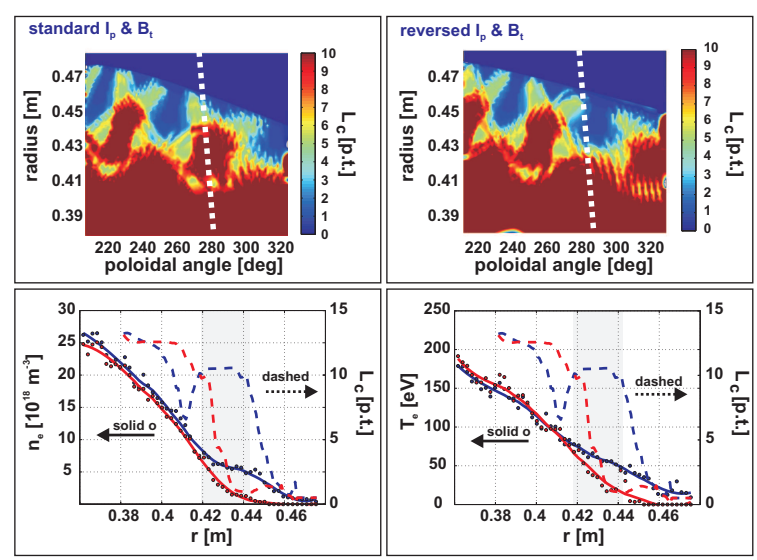

Figure 11: Profile changes in in $m / n=$ $6 / 2$ base mode configuration at poloidal up position: upper row shows the $L_{c}(r, \vartheta)$ for standard $I_{P}$ and $B_{t}$ setting (left column) and reversed $I_{P}$ and $B_{t}$ setting (right column). In lower $n_{e}(r)$ (left column) and $T_{e}(r)$ (right column) from edge Thomson scattering are shown. The profiles were extracted in standard $I_{p} / B_{t}$ configuration (blue profiles, $\circ$ marker) and in reversed $I_{p} / B_{t}$ configuration (red profiles, $\circ$ marker), both with $I_{D E D}=$ $3.0 k A$ applied 

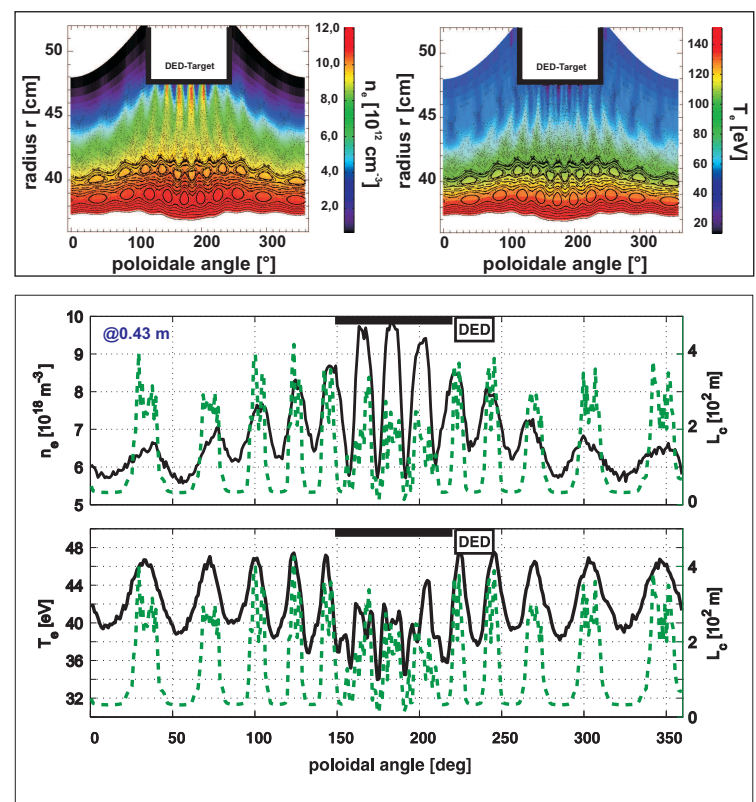

Figure 12: Modeled plasma structure in $m / n=12 / 4$ base mode for TEXTOR discharge \#95895. Upper plots left shows $n_{e}(r, \vartheta)$, upper right plot shows $T_{e}(r, \vartheta)$ with superimposed Poincaré plot. Lower plot shows $n_{e}(\vartheta)$ and $T_{e}(\vartheta)$ as poloidal cuts at $r=0.43 \mathrm{~m}$ (a cut along an formerly unperturbed flux surface is shown). 

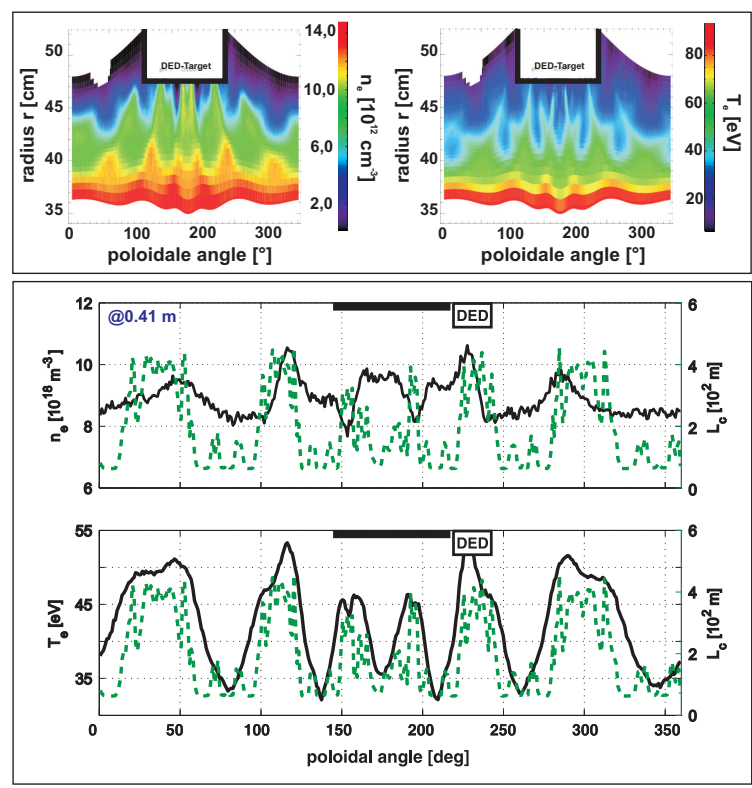

Figure 13: Modeled plasma structure in $m / n=6 / 2$ base mode based on equilibrium of TEXTOR discharge \#95895. Upper plots left shows $n_{e}(r, \vartheta)$, upper right plot shows $T_{e}(r, \vartheta)$ with superimposed Poincaré plot. Lower plot shows $n_{e}(\vartheta)$ and $T_{e}(\vartheta)$ as poloidal cuts at $r=0.41 \mathrm{~m}$ is shown (a cut along an formerly unperturbed flux surface is shown).

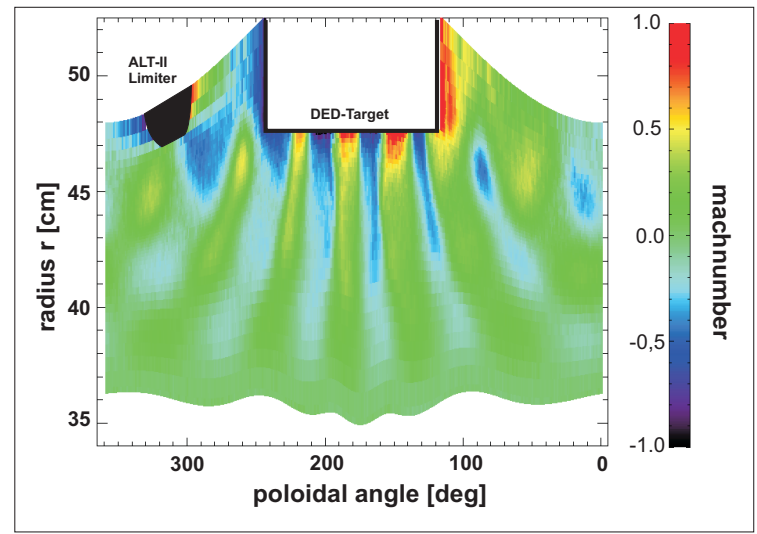

Figure 14: Modeled plasma flow pattern as the mach number distribution $M(r, \vartheta)$ in $m / n=6 / 2$ base mode based on equilibrium of TEXTOR discharge \#95895. 


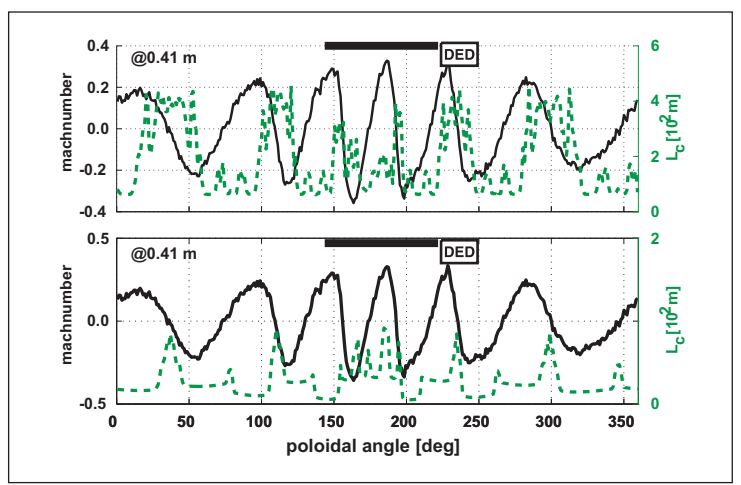

Figure 15: Poloidal profiles of plasma flow pattern $M(\vartheta)$ compared to $L_{c}(\vartheta)$ (upper plot) and shortest $L_{c}(\vartheta)$ (lower plot) cuts at $r=0.41 \mathrm{~m}$

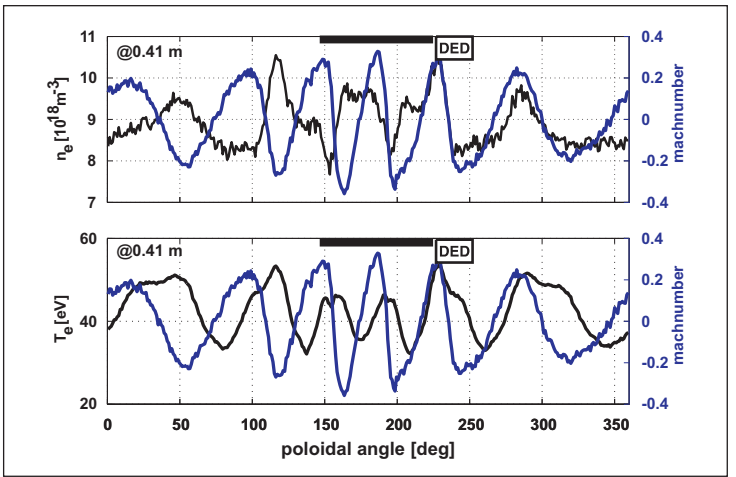

Figure 16: Poloidal profiles of plasma flow pattern $M(\vartheta)$ compared to $n_{e}(\vartheta)$ and $T_{e}(\vartheta)$ cuts at $r=0.41 \mathrm{~m}$ discussed in figure 13 


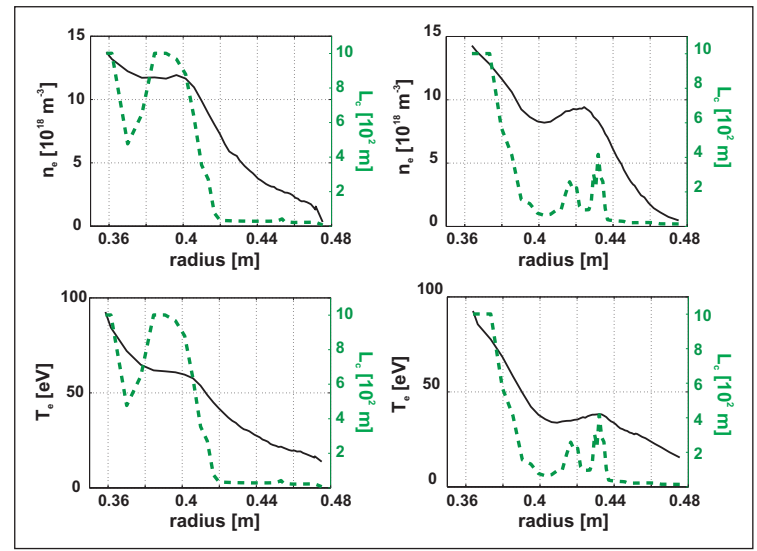

Figure 17: Comparison of modeled $n_{e}(r)$ and $T_{e}(r)$ profiles and the connection length $L_{c}(r)$ from the modeling grid. The poloidal positions for these profiles are shown in figure $13(\mathrm{~m} / \mathrm{n}=6 / 2$ base mode based on equilibrium of TEXTOR discharge \#95895).

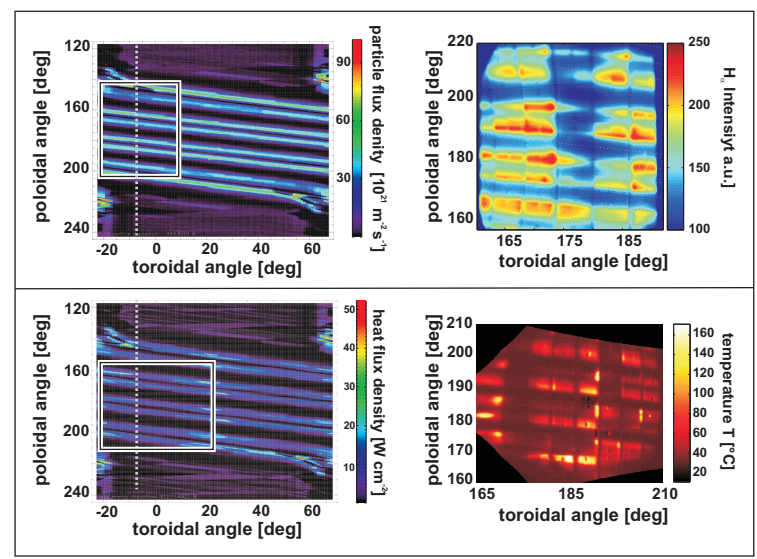

Figure 18: Comparison of modeled and measured deposition pattern on the DED target. Upper row shows the particle flux density $\Gamma_{\perp}(\varphi, \vartheta)$ while lower row shows the heat flux density $q_{\perp}(\varphi, \vartheta)$, both from modeling (left figures) and from experiment (right figures). 


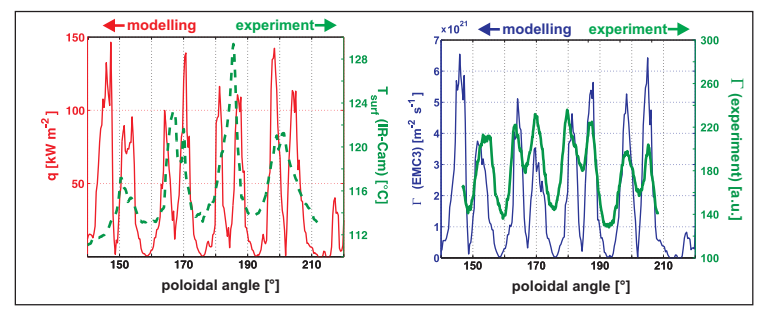

Figure 19: Comparison of modeled and measured heat and particle deposition on the DED target along the poloidal cut depicted as grey dashed line in figure 18. Right figure shows $\Gamma_{\perp}(\vartheta)$ from modeling (blue curve, left abscissa) and from experiment (green curve, right abscissa) while left figure shows $q_{\perp}(\vartheta)$ from modeling (blue curve, left abscissa) and from experiment (green curve, right abscissa). 


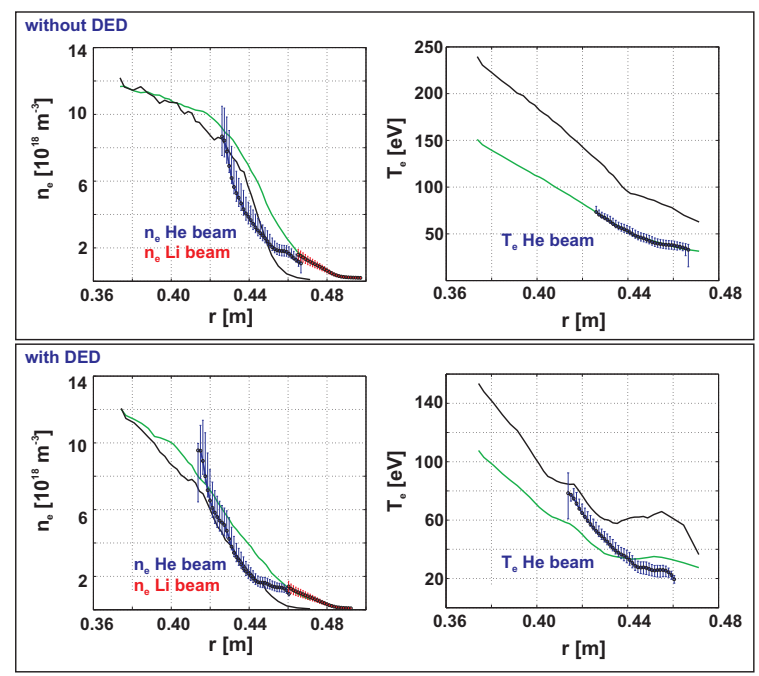

Figure 20: Comparison of modeled and measured $n_{e}(r)$ and $T_{e}(r)$ profiles. Upper figures show results from a reference discharge without DED perturbation applied (\#95896), lower plots shows profile comparison with $I_{D E D}=13.5 \mathrm{kA}(\# 95895)$. Green profiles are from modeling 1 with $D_{\perp}=1.4 \mathrm{~m}^{2} \mathrm{~s}^{-1}$ and $\chi_{\perp}=4.1 \mathrm{~m}^{2} \mathrm{~s}^{-1}$ and black profiles are results from modeling with reduced transport coefficients (modeling 2, $\left.D_{\perp}=0.5 \mathrm{~m}^{2} \mathrm{~s}^{-1}, \chi_{\perp}=3.0 \mathrm{~m}^{2} \mathrm{~s}^{-1}\right)$. Experimental profiles are with error bars, blue profiles from He beam measurements and red profiles form supporting $n_{e}(r)$ measurements with a thermal Li beam.

\section{References}

[1] Masuzaki S. et al., Nuclear Fusion 42, 750 (2002).

[2] Feng Y. et al., this confercence.

[3] König R. et al., Plasma Physics and Controlled Fusion 44, 2365 (2002).

[4] Morisaki T. et al., Journal of Nucl. Mater. 337-339, 154 (2005).

[5] Zohm H., Plasma Physics and Controlled Fusion 38, 105 (1996).

[6] Federici G. et al., Plasma Physics and Controlled Fusion 45, 1523 (2003).

[7] Evans T.E. et al., Nature of Physics 2, 419 (2006).

[8] Liang Y. et. al., Phys. Rev. Lett. 98 (2007).

[9] Fenstermacher M. et al., Journal of Nucl. Mater. 363-365, 476 (2007).

[10] Finken K.H. (Editor), The Dynamic Ergodic Divertor (DED) for TEXTOR-94 (Forschungszentrum Jülich GmbH, 1996), report des fzj, jül-3285 edn.

[11] Schweer B. et al., Journ. Nucl. Mater. 266-269, 673 (1999).

[12] van der Meiden H. J. et al. (AIP, 2006), vol. 77, p. 10E512. 
[13] Park H. et al., Rev. Sci. Instrum. 74, 4239 (2003).

[14] Classen I. et al., Europhysics conference abstracts 29C (2005).

[15] Finken K.H. et al., The structure of magnetic field in the TEXTOR-DED (Schriften des Forschungszentrums Jülich, Band 45, ISBN 3-89336-418-8, 2005).

[16] Eich T. et al., Nuclear Fusion 40, 1757 (2000).

[17] Kobayashi M. et al., Nuclear Fusion 44, S64 (2004).

[18] Rechester A.B. and M.N. Rosenbluth, Phys. Rev. Let. 40 (1978).

[19] Evans T.E. et al., Journal of Nucl. Mater. 145-147, 812 (1987).

[20] Evans T.E. et al., Journal of Nucl. Mater. 162-164 (1989).

[21] Ghendrih Ph. et al., Nuclear Fusion 42, 1221 (2002).

[22] Nguyen F. et al., Nuclear Fusion 37, 743 (1997).

[23] Abdullaev SS. et al., Nuclear Fusion 43, 299 (2003).

[24] Jakubowski M.W. et al., AIP conference proceedings CP812, 195 (2006).

[25] Tokar' M.Z. et al., Plasma Physics and Controlled Fusion 39, 569 (1997).

[26] Samain A. et al., Phys. Fluids B 5 (1993).

[27] Lehnen M. et al., Plasma Physics and Controlled Fusion 47, B237 (2005).

[28] Jakubowski M.W., O. Schmitz et al., Phys. Rev. Lett. 96 (2006).

[29] Schmitz O. et al., Europhysics conference abstracts 29C (2005).

[30] Lehnen M. et al., Journal of Nucl. Mater. 363-365, 377 (2007).

[31] Jakubowski M.W. et al., Nuclear Fusion 44, S1 (2004).

[32] Schmitz O., D. Harting et al., Journal of Nucl. Mater. 363-365, 680 (2007).

[33] Lehnen M. et al., Journal of Nucl. Mater. 337-339, 171 (2005).

[34] Reiter D. et al., Fusion Science and Technology 47, 172 (2005).

[35] Harting D. et al., submitted to Plasma Physics and Controlled Fusion (2007).

[36] Stangeby P.C., The plasma boundary of magnetic fusion devices (Plasma Physics Series, IoP Publishing LtD, Bristol, UK, 2000). 